\title{
17. ORIGIN OF DIAGENETIC CARBONATE MINERALS RECOVERED FROM THE NEW JERSEY CONTINENTAL SLOPE ${ }^{1}$
}

\author{
Karen S. Hicks, ${ }^{2,3}$ John S. Compton, ${ }^{2}$ Stuart McCracken, ${ }^{4}$ and Adam Vecsei ${ }^{5}$
}

\begin{abstract}
Diagenetic carbonate minerals in upper Cenozoic sediments recovered from the New Jersey slope are a byproduct of organic matter degradation and the dissolution of precursor biogenic calcite, mostly foraminifers and nannofossils. The diagenetic carbonate minerals include dolomite, siderite, and calcite. Dolomite occurs as disseminated rhombs and cemented beds and nodules. Disseminated dolomite rhombs up to $10 \mu \mathrm{m}$ in diameter are currently forming in the uppermost $200 \mathrm{~m}$ of sediment as indicated by the exponential decrease in pore-water $\mathrm{Mg}$ and $\mathrm{Ca}$. The dolomite is both calcian and ferroan with an average composition of $(\mathrm{Ca})_{1.12}(\mathrm{Mg})_{0.8}(\mathrm{Fe})_{0.08}\left(\mathrm{CO}_{3}\right)_{2}$. Carbon isotopic values of the dolomite-cemented nodules that occur at greater stratigraphic depths range from $-15.0 \%$ to $+4.5 \%$ and indicate dolomitization in the sulfate-reduction and the upper methanogenesis zones. Organic matter degradation by way of sulfate reduction promotes precipitation of dolomite by removing sulfate ion and increasing the carbonate alkalinity.

Siderite occurs as poorly cemented layers and nodules with an average composition of $(\mathrm{Fe})_{0.72}(\mathrm{Mg})_{0.20}(\mathrm{Ca})_{0.08} \mathrm{CO}_{3}$. Carbon isotopic values for siderite range from $-2.0 \%$ to $+11.3 \%$, indicating formation in the methanogenesis zone. Precipitation of siderite and ferroan dolomite is consistent with the large amount of Fe-rich terrigenous siliciclastics in these sediments and high pore-water $\mathrm{Fe}$ concentrations. Diagenetic dolomite and calcite are favored over siderite during early burial because pore-water $\mathrm{Fe}$ is removed by the precipitation of glaucony in the oxic to suboxic zones and iron sulfide minerals in the sulfate-reduction zone.

Diagenetic calcite is less common, occurring as cemented nodules with an average composition of $(\mathrm{Ca})_{0.91}(\mathrm{Mg})_{0.07}$ $(\mathrm{Fe})_{0.02} \mathrm{CO}_{3}$. Diagenetic calcites have carbon isotopic compositions that range from $-0.8 \%$ to $-37 \%$. The very low carbon isotopic values of some calcite cements suggest that they formed during methane oxidation near the sediment/seawater interface where the relatively high seawater sulfate concentration and low $\mathrm{Mg} / \mathrm{Ca}$ ratio favor calcite rather than dolomite precipitation. ${ }^{87} \mathrm{Sr} /{ }^{86} \mathrm{Sr}$-derived ages of the diagenetic carbonates generally do not coincide with the biostratigraphic age of the sediment. The availability of precursor carbonate and variations in sedimentation rate related to sea-level fluctuations appear to be the primary factors that determine the abundance and mineralogy of the diagenetic carbonates.
\end{abstract}

\section{INTRODUCTION}

The primary objective of Ocean Drilling Program (ODP) Leg 150 was to determine the age of major unconformities and to evaluate their correlation with glacioeustatic age estimates obtained from the $\delta^{18} \mathrm{O}$ record. Leg 150 drilled the upper to middle continental slope and the uppermost continental rise of the New Jersey Margin (Fig. 1). This margin is characterized by highly variable sedimentation rates, as well as terrigenous and organic carbon contents. In organic-rich sediments, bacterial degradation of organic matter can significantly alter pore-water chemistry and result in the precipitation of early diagenetic carbonate, fluorapatite (phosphorite) and pyrite (Compton, 1988a). These minerals are termed organogenic because their precipitation is a direct result of organic matter diagenesis. The degradation of organic matter takes place within a succession of microbial depth zones: oxic, suboxic (Mn and $\mathrm{Fe}$ oxide reduction), sulfate reduction, methanogenesis, and fermentation, followed by thermal degradation (Claypool and Kaplan, 1974; Froelich et al., 1979).

Diagenetic dolomite is common in continental margin sequences which have sedimentation rates of less than $500 \mathrm{~m} / \mathrm{m}$.y. and are or-

'Mountain, G.S., Miller, K.G., Blum, P., Poag, C.W., and Twichell, D.C. (Eds.), 1996. Proc. ODP, Sci. Results, 150: College Station, TX (Ocean Drilling Program).

${ }^{2}$ Department of Marine Science, University of South Florida, St. Petersburg, FL 33701-5016, U.S.A. compton@marine.usf.edu

'Present address: 2665 Sequoia Terrace, Palm Harbor, FL 34683, U.S.A.

${ }^{4}$ Department of Geology, University of Western Australia, Nedlands, Western Australia 6009. Australia.

${ }^{5}$ Geologisches Institut der Universtät, Albertstrasse 23B, D-79104 Freiburg i.Br., Federal Republic of Germany. ganic rich ( $>0.5 \mathrm{wt} \%$ organic carbon) (Baker and Burns, 1985). Other carbonate minerals, such as calcite, siderite, and rhodochrosite, may also form depending on the availability of $\mathrm{Mg}, \mathrm{Ca}, \mathrm{Fe}$, and $\mathrm{Mn}$ (e.g., Hein and Koski, 1987). For example, methane-derived carbonate cements are common in rapidly deposited, organic-rich sediments where methane seeps migrate to the sediment/seawater interface (e.g., Iverson and Jørgensen, 1985; Hovland et al., 1987; Paull et al., 1992). Dolomite can be detrital, reworked from upslope shallowwater environments or form in place (authigenic). Organogenic dolomite is distinguished from other types of sedimentary dolomites by having a significant portion of its carbon derived from degradation of organic matter as well as the dissolution of biogenic calcite (Spotts and Silverman, 1966). Pore waters can become highly supersaturated with respect to diagenetic carbonate minerals from an increase in carbonate alkalinity associated with the bacterial degradation of organic matter (Compton, 1988b). $\mathrm{Mg}$ and $\mathrm{Ca}$ required during dolomite precipitation are supplied by diffusion from overlying seawater (Baker and Burns, 1985; Compton and Siever, 1986). The presence of a carbonate precursor enhances dolomitization by providing an additional source of $\mathrm{Ca}$ and carbonate ions, because the diffusion of $\mathrm{Ca}$ from overlying seawater is limited by the significantly lower seawater concentration of $\mathrm{Ca}$ compared to $\mathrm{Mg}$.

Early diagenetic precipitation of siderite occurs in two distinct geochemical environments: the slightly reducing conditions of the suboxic zone, and the strongly reducing conditions of the methanogenesis zone. Suboxic conditions (microbial iron reduction) are favored in marine environments that have a relatively low concentration of organic matter, slow sedimentation rates (Berner, 1981; Coleman, 1985), and an Eh too high to allow bacterial sulfate-reduction and pyrite formation (Maynard, 1982; Mozley and Carothers, 1992). 
Figure 1. Location of the Leg 150 drill sites, DSDP Legs 11 and 95 drill sites, and onshore boreholes on the New Jersey continental margin.

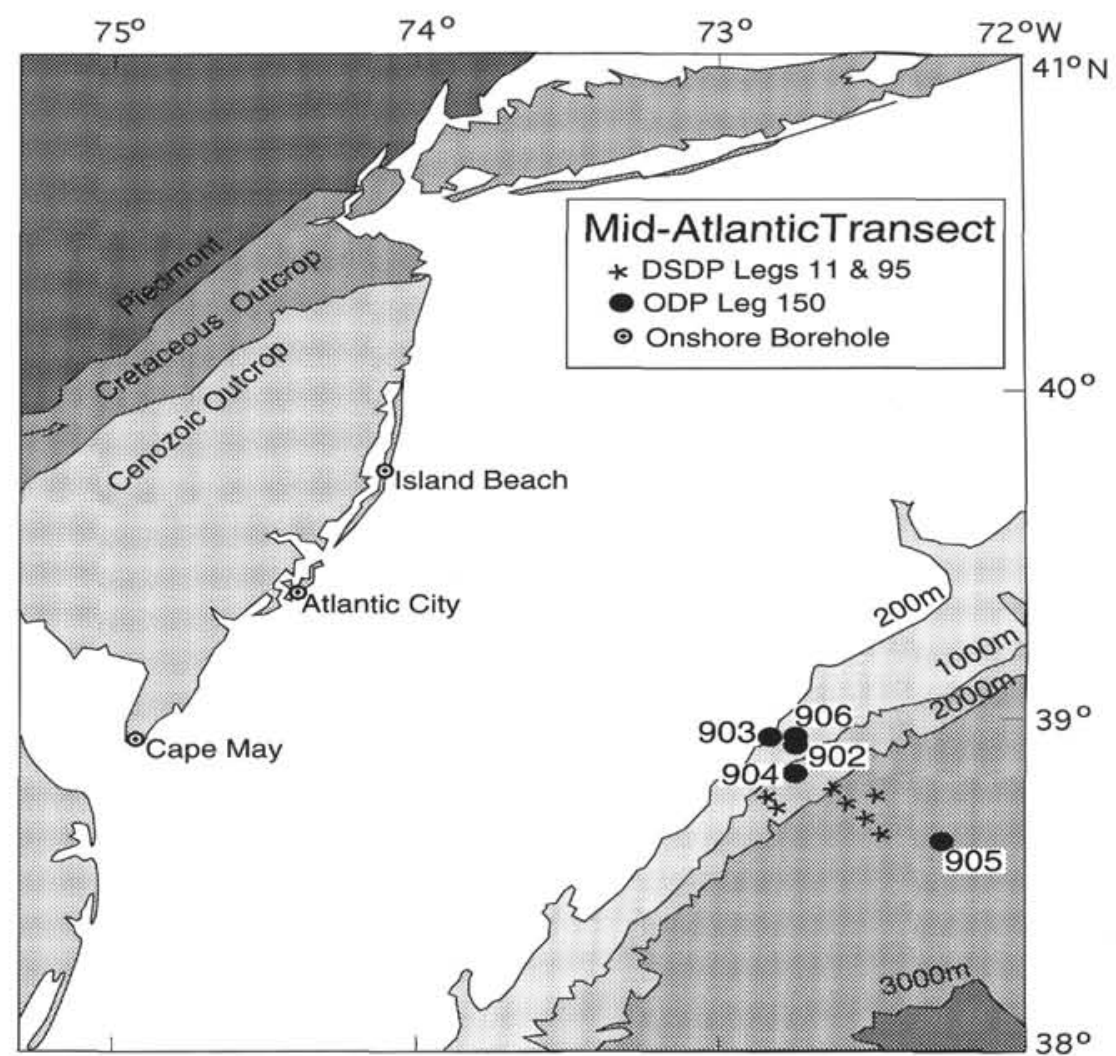

Siderite can also form in nearshore and intertidal sediments that are subject to alternating anoxic and oxic conditions (Coleman, 1985). The precipitation of siderite in the methanogenesis zone has been observed in a variety of continental (e.g., estuarine) and marine sediments (Moore et al., 1992; Pye et al., 1990; Thyne and Gwinn, 1994). The methanogenesis zone is favored in environments characterized by low sulfate concentrations, high sedimentation rates, and high organic carbon content (Coleman, 1985; Mozley and Carothers, 1992). Geochemical environments conducive to siderite formation require high bicarbonate concentrations and high $\mathrm{Fe}^{2+}$ concentrations. Siderite exhibits a wide range of compositions, dependent upon pore-water chemistry, with major substitution of $\mathrm{Mg}^{2+}, \mathrm{Ca}^{2+}$, and $\mathrm{Mn}^{2+}$ (Curtis et al., 1975; Curtis and Coleman, 1986). However, the precise porewater $\mathrm{Fe} /(\mathrm{Ca}+\mathrm{Mg})$ ion ratio required for siderite precipitation is unknown.

In this paper, the origin of diagenetic carbonates recovered from the New Jersey Margin is developed by integrating pore-water chemistry and carbonate petrology, mineralogy, and elemental and isotopic composition. There are few studies of late Cenozoic siderite formation. Sediment recovered during Leg 150 provides a unique opportunity to study diagenetic siderite formation in the context of pore-water analysis and a well-defined stratigraphic framework.

\section{METHODS}

Recovered carbonate cemented intervals were sampled, washed, and powdered in an agate mortar and pestle. Mineralogy of the bulk powders was determined by X-ray diffraction (XRD) using $\mathrm{Cu} \mathrm{K} \alpha$ radiation on a Scintag XDS 2000 instrument at the University of South Florida (USF). Small rock chips were examined on an ISIDS130 scanning electron microscope (SEM) at USF. Samples that contained a mixture of dolomite and calcite or siderite and calcite, on the basis of the XRD results, were treated with $\mathrm{Na}$-acetate/acetic acid solution buffered to $\mathrm{pH} 5(\mathrm{NaOAc})$ to remove calcite preferentially. After NaOAc treatment, removal of calcite was confirmed by XRD analysis. Homogenous splits of treated and untreated bulk powders $(250-500 \mathrm{mg})$ were digested in $10 \% \mathrm{HCl}$ at room temperature and $\mathrm{el}-$ emental composition of the acid-soluble fraction was determined by $\mathrm{X}$-ray Assay Laboratories (Toronto, Ontario, Canada) using inductively coupled plasma (ICP) emission spectroscopy.

Stable carbon and oxygen isotopic compositions of the powdered diagenetic carbonate samples were measured using a Finnigan Mat 250 isotope mass spectrometer at USF and at the University of Tasmania. At USF, the bulk powdered samples were placed in glass tubes, evacuated and baked for $1 \mathrm{hr}$ at $380^{\circ} \mathrm{C}$ to convert organic carbon to an inert form. The samples were then placed in a side-arm reaction vessel in a $25^{\circ} \mathrm{C}$ water bath and allowed to react with $100 \%$ $\mathrm{H}_{3} \mathrm{PO}_{4}$ until the reaction was complete. Dolomite and calcite reaction time was $24-48 \mathrm{hr}$; siderite required at least 7 days to yield sufficient $\mathrm{CO}_{2}$ for analysis. Samples at the University of Tasmania were reacted with $100 \% \mathrm{H}_{3} \mathrm{PO}_{4}$ in a $50^{\circ} \mathrm{C}$ water bath. The $\mathrm{CO}_{2}$ yield apparently does not affect the $\delta^{13} \mathrm{C}$ value of siderite; however, yields less than $80 \%$ were found to decrease the $\delta^{18} \mathrm{O}$ values of siderite (Carothers et al., 1988). All uncorrected $\delta^{18} \mathrm{O}$ values were calculated using a fractionation factor for phosphoric acid liberated- $\mathrm{CO}_{2}$ and calcite at $25^{\circ} \mathrm{C}$ of 1.01020 (McCrea, 1950). Dolomite $\delta^{18} \mathrm{O}$ values were corrected by subtracting $0.8 \%$ using the fractionation factor estimated for the phosphoric acid liberated $\mathrm{CO}_{2}$-dolomite reaction at $25^{\circ} \mathrm{C}$ of 1.01100 (Sharma and Clayton, 1965). The siderite $\delta^{18} \mathrm{O}$ values were corrected by subtracting $1.45 \%$ using the fractionation factor for the phosphoric acid liberated $\mathrm{CO}_{2}$-siderite reaction at $25^{\circ} \mathrm{C}$ of 1.01165 (Carothers et al., 1988). Siderites from Leg 150 have substantial amounts of $\mathrm{Mg}$ and $\mathrm{Ca}$ substitution and, therefore, their fractionation factor may be slightly different than that determined by Carothers et al. (1988) for pure siderite $\left(\mathrm{FeCO}_{3}\right)$. The $\delta^{18} \mathrm{O}$ data from the University of Tasmania were adjusted by subtracting 1 per mil $(0.04 \%$ oper degree $)$ to correct for the higher reaction temperature. 
Sr-isotopic analyses were performed on homogenous carbonate bulk powders at Rutgers University. The carbonate was dissolved in $1.5 \mathrm{~N} \mathrm{HCl}$ and in acetate/acetic acid buffer. Standard ion-exchange techniques were used to separate strontium for analysis on a VG Sector mass spectrometer. Internal precision on the Sector is approximately \pm 0.000011 and external precision (interrun variability) is \pm 0.000030 or better; the NBS standard is routinely measured at Rutgers as 0.710250 (Miller et al., 1988). Ages were estimated from the Sr-isotopic measurements using the empirical age $-{ }^{87} \mathrm{Sr} /{ }^{86} \mathrm{Sr}$ relationship established at Deep Sea Drilling Project (DSDP) Site 522 (Miller et al., 1988); this linear relationship is apparently valid from the late Eocene $(\sim 38 \mathrm{Ma})$ to the early Miocene ( 22 Ma). Given the rate of change of ${ }^{87} \mathrm{Sr} /{ }^{86} \mathrm{Sr}$ and the external precision, the stratigraphic resolution of this interval is $\pm 1 \mathrm{~m} . y$. or better (Miller et al., 1988). Miocene ages were derived from two linear relationships established at Site 747 (Oslick et al., 1994). The lower Miocene (23-15.5 Ma) age estimates have age errors of \pm 0.7 m.y. and the middle Miocene (15.2-9.9 Ma) have age errors of \pm 1.4 m.y. (Oslick et al., 1994). All ages are reported to the time scale of Berggren et al. (1995).

Squeezed pore-water samples were analyzed on board the JOIDES Resolution using standard techniques (Mountain, Miller, Blum, et al., 1994). Pore-water Fe and K were also measured on a direct-current plasma emission spectrometer (DCP) at USF.

\section{RESULTS}

Carbonate cemented nodules and layers were recovered from Sites 902, 903, 905, and 906 (Fig. 1). Although disseminated carbonate occurs at Site 904, no cemented carbonate nodules were recovered. A single, large dolostone interval was recovered at Site 905 at $574.9 \mathrm{~m}$ below seafloor (mbsf) (Sample 150-905A-64X-5, 65-68 $\mathrm{cm}$ ). Site 905 was drilled in approximately $2700 \mathrm{~m}$ water depth on the continental rise. Site 902 was drilled in approximately $800 \mathrm{~m}$ water depth on the upper continental slope. The majority of the carbonate nodules were recovered from Site 903 on the upper continental slope drilled in approximately $445 \mathrm{~m}$ water depth, $4.4 \mathrm{~km}$ upslope of Site 902. Site 906 was drilled at approximately $925 \mathrm{~m}$ depth on the middle continental slope, $3.3 \mathrm{~km}$ north of Site 902.

Carbonate nodules and cemented layers were recovered from upper Oligocene to upper Miocene silty clay sediment (Fig. 2). Disseminated diagenetic carbonate was recovered from Pleistocene sediment. Siderite nodules commonly occur directly below or above glaucony-rich intervals and are most abundant in middle Miocene sediment. Dolomite and calcite nodules occur both within and outside of glaucony-rich intervals.

\section{Petrology}

Dolomite rhombs, 0.1 to $10 \mu \mathrm{m}$ in size, are observed to grow within foraminifer tests and replace nannofossils (PI. 1). Dolomite occurs as tightly cemented light to dark grey layers and nodules. Dolomite is primarily a pore-filling cement. Dolomite and calcite nodules from glaucony-rich intervals contain abundant glaucony and quartz sand size grains. In Sample 150-903A-41X-1, 89-94 cm, a calcite cemented nodule containing glauconitic grains shows two phases of calcite cementation. The original precipitation of calcite was followed by an infilling of calcite cement in shrinkage cracks and around glauconitic grains. Unlike siderite, both the calcite and dolomite nodules contain abundant framboidal pyrite. Siderite occurs as poorly cemented (friable) cream colored layers and nodules. Siderite rhombs, similar to the dolomite rhombs, are observed to grow within foraminifer tests and replace nannofossils (Pl. 2).

\section{Mineralogy and Elemental Composition}

Compositional fields are well defined for most calcite, dolomite, and siderite samples (Tables 1, 2; Fig. 3). Samples that fall outside these fields contain a mixture of carbonate minerals or, in the case of Sample 150-902D-48X-3, 7-9 cm, a mixture of siderite and francolite. The siderite/francolite sample falls in the siderite field after subtraction of the francolite calcium content. Dolomite ranges from nearly stoichiometric to calcian and ferroan with a total of 14 samples having an average composition of $(\mathrm{Ca})_{1.12}(\mathrm{Mg})_{0.80}(\mathrm{Fe})_{0.08}\left(\mathrm{CO}_{3}\right)_{2}$. A total of 20 siderite samples have an average composition of $(\mathrm{Fe})_{0.72}(\mathrm{Mg})_{0.20}(\mathrm{Ca})_{0.08} \mathrm{CO}_{3}$. The amount of $\mathrm{Ca}$ substitution in New Jersey Margin siderites is fairly constant, ranging from 6 to $13 \mathrm{~mol} \%$ with the majority of siderite containing 6 to $8 \mathrm{~mol} \%$. The amount of $\mathrm{Mg}$ substitution is more variable and ranges from 12 to $32 \mathrm{~mol} \% \mathrm{Mg}$ (Table 2). The Mn content of the siderite is low, ranging from 1000 to $2040 \mathrm{ppm}$. The average composition of nine diagenetic calcite samples is $(\mathrm{Ca})_{0.91}(\mathrm{Mg})_{0.07}(\mathrm{Fe})_{0.02} \mathrm{CO}_{3}$.

$\mathrm{Al}$ and $\mathrm{K}$ show weak negative correlations with carbonate content, and $\mathrm{Zn}$ shows a negative correlation with siderite and calcite (Fig. 4). Be, Mn, and V show a positive correlation with siderite content but not with dolomite or calcite. Ba content tends to increase with increasing siderite, dolomite, and calcite. Dolomite and calcite have highly variable $\mathrm{Sr}$ contents that are consistently higher than the $\mathrm{Sr}$ content of siderite.

\section{Stable Isotopes}

Carbon isotopic values for dolomite range from $-15.0 \%$ to $+4.5 \%$ (PDB) and corrected (subtraction of $0.8 \%$ ) $\delta^{18} \mathrm{O}$ values range from $-8.1 \%$ to $+5.1 \%$ (PDB) (Table 3 ; Fig. 5 ). Siderite $\delta^{13} \mathrm{C}$ values range from $-2.0 \%$ to $+11.3 \%$ (PDB) and corrected (subtraction of $1.45 \%$ ) $\delta^{18} \mathrm{O}$ values of siderite range from $-1.4 \%$ to $+6.1 \%$ (PDB). The $\delta^{13} \mathrm{C}$ values of calcite nodules range from $-37 \%$ to $+0.6 \%$ o (PDB) and $\delta^{18} \mathrm{O}$ values range from $-1.3 \%$ to $+4.3 \%$ (PDB) (Fig. 5). The $\delta^{18} \mathrm{O}$ values of the diagenetic carbonates tend to decrease with increasing burial depth (Fig. 6). The $\delta^{13} \mathrm{C}$ values of the diagenetic carbonates show broad shifts with increasing burial depths (Fig. 7).

\section{ORIGIN OF DIAGENETIC CARBONATES}

Pore waters from Neogene sediments recovered from the New Jersey Margin exhibit an exponential decrease in sulfate ion concentration, typical of marine sediment undergoing organic matter degradation by sulfate-reducing bacteria (Claypool and Kaplan, 1974; Gieskes, 1983). Organic matter degradation by sulfate reduction results in an increase in pore-water phosphate, ammonium, and carbonate alkalinity (Table 4; Fig. 8) that can lead to the precipitation of diagenetic phosphate, sulfide, and carbonate minerals. Differences in mineralogy (dolomite, siderite, and calcite) and $\delta^{13} \mathrm{C}$ signatures of the diagenetic carbonates depend on the pore-water $\mathrm{Fe}, \mathrm{Mg}$, and $\mathrm{Ca}$ concentrations and the rate of organic matter degradation, which controls the sediment depths of the microbial sulfate reduction and methanogenesis zones (Berner, 1980; Mozley and Burns, 1993).

\section{Dolomite}

Removal of $\mathrm{Ca}$ and $\mathrm{Mg}$ ions, evident in the pore-water profiles (Fig. 9), indicates that dolomite is currently precipitating in the uppermost $200 \mathrm{mbsf}$ at the upper slope sites on the New Jersey Margin. The presence of $1-10-\mu \mathrm{m}$ disseminated dolomite rhombs was confirmed by XRD and SEM (PI. 1). Several factors that enhance dolomite precipitation at low temperatures on the New Jersey Margin include: (1) high degree of supersaturation from an increase in carbonate alkalinity that results from bacterial degradation of organic matter (Claypool and Kaplan, 1974; Compton, 1988b); (2) high pore-water $\mathrm{Mg} / \mathrm{Ca}$ ratios up to twice that of seawater (Fig. 9); (3) removal of sulfate ion, an inhibitor of dolomitization (Baker and Kastner, 1981); and (4) the presence of precursor carbonate (foraminifers and nannofossils) (Compton, 1992). 

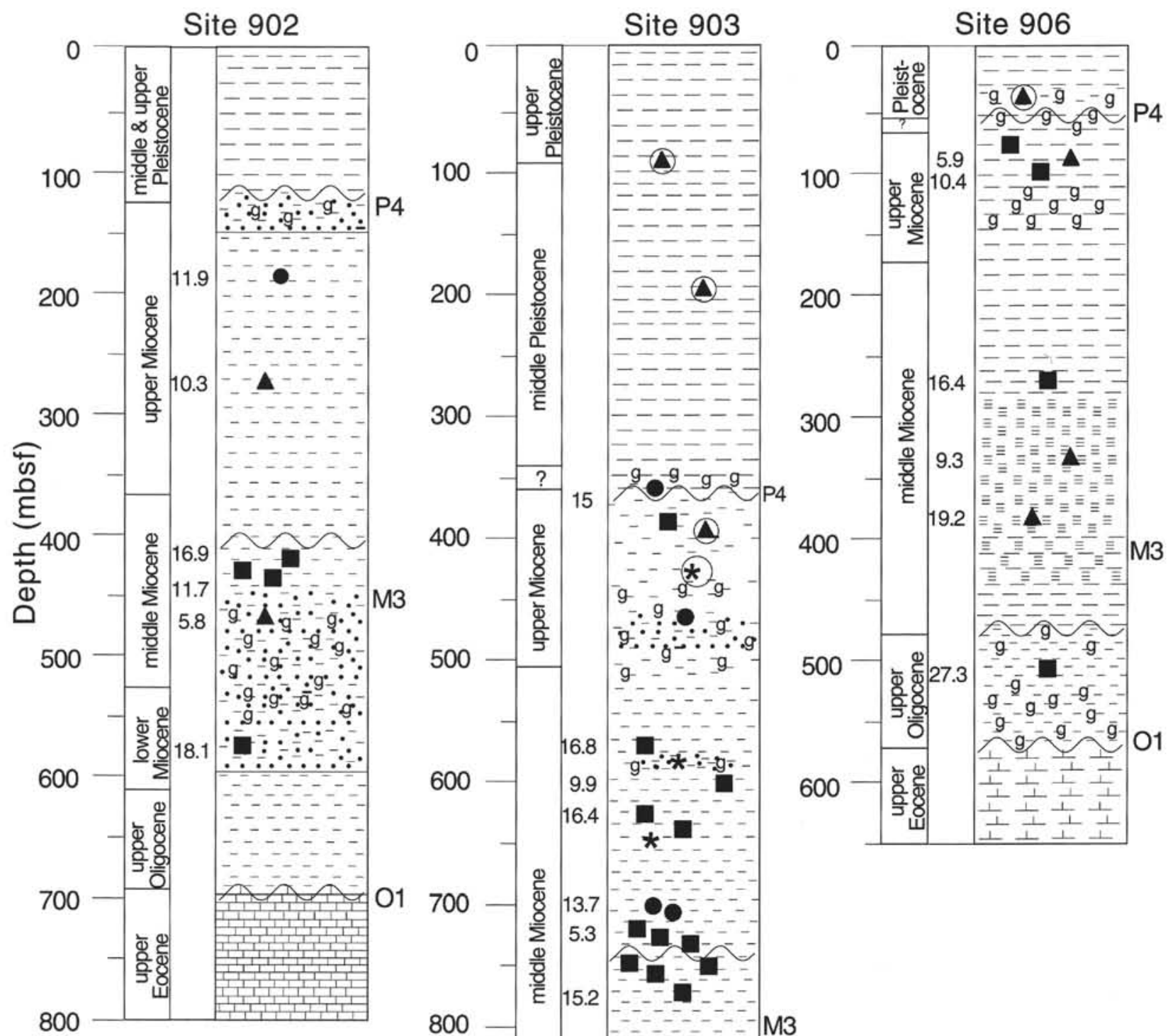

800
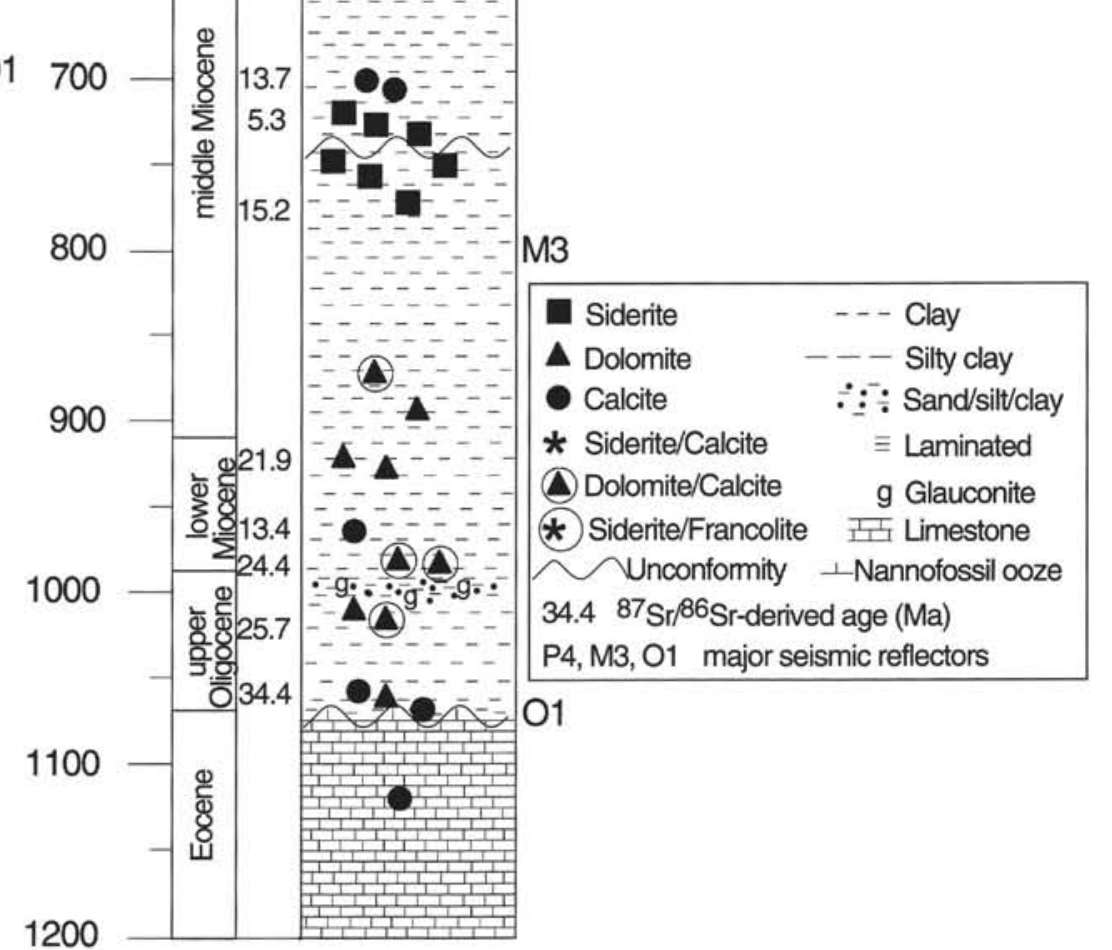

Figure 2. Stratigraphic columns depicting major lithologic variations at Sites 902,903 , and 906 . The sampled diagenetic carbonate nodules are shown by their mineralogy. The Sr-derived ages of the diagenetic carbonate samples are shown to the left of the columns and the major seismic reflectors (01, M3, and P4) are shown to the right of the columns. 
Table 1. Mineralogy and ICP elemental analysis of the acid-soluble fraction of bulk diagenetic carbonate samples from Leg 150.

\begin{tabular}{|c|c|c|c|c|c|c|c|c|c|c|c|c|c|c|c|c|c|}
\hline $\begin{array}{l}\text { Core, section, } \\
\text { interval (cm) }\end{array}$ & $\begin{array}{l}\text { Depth } \\
\text { (mbsf) }\end{array}$ & Mineralogy & $\underset{(\mathrm{wt} \%)}{\mathrm{Ca}}$ & $\underset{(\mathrm{wt} \%)}{\mathrm{Mg}}$ & $\underset{(\mathrm{wt} \%)}{\mathrm{Fe}}$ & $\begin{array}{l}\text { Carbonate } \\
\text { (wt \%) }\end{array}$ & $\begin{array}{c}\mathrm{Mn} \\
(\mathrm{ppm})\end{array}$ & $\underset{(\mathrm{ppm})}{\mathrm{Sr}}$ & $\begin{array}{c}\mathrm{Ba} \\
(\mathrm{ppm})\end{array}$ & $\underset{(\mathrm{wt} \%)}{\mathrm{Na}}$ & $\underset{(w t \%)}{\mathrm{K}}$ & $\underset{(\mathrm{w} t \%)}{\mathrm{Al}}$ & $\underset{(w t \%)}{P}$ & $\begin{array}{c}\mathrm{V} \\
(\mathrm{ppm})\end{array}$ & $\begin{array}{c}\mathrm{Zn} \\
(\mathrm{ppm})\end{array}$ & $\underset{(\mathrm{ppm})}{\mathrm{Zr}}$ & $\begin{array}{c}\mathrm{Be} \\
(\mathrm{ppm})\end{array}$ \\
\hline \multicolumn{18}{|l|}{ 150-902D- } \\
\hline $21 X-3,121-126$ & 174.24 & Calcite & 23.5 & 1.2 & 0.3 & 63.3 & 82 & 820 & 193 & 0.3 & 0.1 & 0.1 & 0.2 & 19 & 25.2 & 20.8 & 1.2 \\
\hline $30 \mathrm{X}-6,13-17$ & 265.33 & Dolomite & 13.7 & 7.2 & 1.0 & 61.3 & 422 & 350 & 83 & 0.2 & 0.1 & 0.2 & 0.1 & 27 & 15.4 & 31.7 & 0.8 \\
\hline $48 X-3,7-9$ & 433.67 & Siderite/francolite & 5.4 & 1.9 & 13.6 & 48.1 & 507 & 1010 & 418 & 0.5 & 0.1 & 0.2 & 2.4 & 59 & 15.8 & 64.1 & 4.4 \\
\hline $48 \mathrm{X}-4,104-102$ & 436.12 & Siderite & 0.5 & 1.0 & 7.4 & 20.1 & 398 & 47.6 & $\begin{array}{r}410 \\
64\end{array}$ & 0.6 & 0.1 & 0.1 & 0.1 & 46 & 34.8 & 4.4 & 1.8 \\
\hline $48 \mathrm{X}-6,16-18$ & 438.26 & Siderite & 0.9 & 1.5 & 14.7 & 38.1 & 510 & 80 & 126 & 0.5 & 0.1 & 0.1 & 0.1 & 77 & 25.4 & 4.8 & 3.0 \\
\hline $51 X-3,65-67$ & 462.55 & Dolomite & 12.8 & 6.8 & 1.5 & 58.8 & 139 & 101 & 25 & 0.2 & 0.1 & 0.1 & 0.1 & 6 & 24.5 & 1.9 & $<0.5$ \\
\hline $63 \mathrm{X}-1,1-5$ & 574.41 & Siderite & 1.8 & 2.3 & 30.0 & 74.7 & 900 & 144 & 216 & 0.2 & 0.0 & 0.1 & 0.3 & 109 & 10.2 & 8.4 & 5.6 \\
\hline \multicolumn{18}{|l|}{$150-903 \mathrm{~A}-$} \\
\hline $11 \mathrm{H}-3,145-150$ (untreated) & 99.45 & Dolomite/calcite & 3.9 & 1.0 & 1.3 & 15.9 & 277 & 73.7 & 20 & 0.2 & 0.2 & 0.3 & 0.1 & 9 & 28.2 & 4.7 & 0.6 \\
\hline $24 \mathrm{X}-3,145-150$ (untreated) & 196.35 & Dolomite/calcite & 3.7 & 1.0 & 1.1 & 15.0 & 162 & 6 & 33 & 0.4 & 0.2 & 0.5 & 0.1 & 12 & 37.5 & 6.0 & $<0.5$ \\
\hline $41 X-1,89-94$ & 356.59 & Calcite & 17.1 & 1.0 & 0.3 & 46.7 & 9 & 306 & 552 & 0.1 & 0.2 & 0.1 & 0.1 & 8 & 6.5 & 1.0 & $<0.5$ \\
\hline $41 X-1,89-94 *$ & 356.59 & Calcite & 19.4 & 1.1 & 0.2 & 52.8 & 10 & 334 & 352 & 0.2 & 0.3 & 0.1 & 0.1 & 7 & 15.7 & 2.7 & $<0.5$ \\
\hline $44 X-5,103-109$ & 391.60 & Siderite & 0.6 & 0.4 & 2.1 & 7.3 & 89 & 35.6 & 154 & 0.3 & 0.4 & 0.4 & 0.0 & 23 & 55.4 & 6.4 & 1.1 \\
\hline $45 \mathrm{X}-2,12-19$ (untreated) & 395.92 & Calcite/dolomite & 14.5 & 2.8 & 2.4 & 50.7 & 194 & 938 & 1250 & 0.3 & 0.1 & 0.2 & 0.4 & 62 & 28.2 & 76.3 & 1.9 \\
\hline $47 \mathrm{X}-2,56-62$ (untreated) & 415.76 & Calcite/siderite & 11.1 & 0.9 & 2.8 & 36.7 & 244 & 525 & 718 & 0.4 & 0.1 & 0.1 & 0.2 & 58 & 26.4 & 66.6 & 1.5 \\
\hline $51 X-3,137-139$ & 456.57 & Calcite & 20.0 & 1.3 & 0.4 & 55.2 & 32 & 769 & 347 & 0.2 & 0.1 & 0.1 & 0.1 & 34 & 14.2 & 25.2 & 1.2 \\
\hline $63 \mathrm{X}-6,148-150$ & 576.78 & Siderite & 0.7 & 1.1 & 8.2 & 22.3 & 256 & 67 & 87 & 0.6 & 0.1 & 0.2 & 0.1 & 80 & 38.4 & 4.7 & 2.0 \\
\hline $64 \mathrm{X}-4,36-38$ (treated) & 582.36 & Siderite & 1.0 & 2.4 & 10.5 & 32.7 & 487 & 60.1 & $\begin{array}{l}01 \\
73\end{array}$ & 0.1 & 0.1 & 0.2 & 0.1 & 91 & 16.1 & 26.1 & 3.1 \\
\hline $69 \mathrm{X}-5,17-19$ & 631.77 & Siderite & 1.1 & 2.0 & 13.5 & 37.8 & 528 & 88.3 & 112 & 0.6 & 0.1 & 0.1 & 0.1 & 98 & 27.6 & 17.1 & 4.0 \\
\hline $70 X-2,76-78$ & 637.46 & Siderite & $\begin{array}{l}1.1 \\
1.2\end{array}$ & 1.8 & 8.2 & 26.2 & 313 & 87.5 & 103 & 0.6 & 0.1 & 0.1 & 0.1 & $\begin{array}{l}78 \\
80\end{array}$ & 27.4 & 7.3 & $\begin{array}{l}4.0 \\
2.0\end{array}$ \\
\hline $71 \mathrm{X}-1,103-106$ (untreated) & 648.71 & Calcite/siderite & 4.6 & 0.5 & 0.8 & 14.7 & 84 & 174 & 167 & 0.8 & 0.3 & 0.3 & 0.1 & 18 & 46.1 & 45.6 & 1.0 \\
\hline \multicolumn{18}{|l|}{$150-903 \mathrm{C}-$} \\
\hline $10 \mathrm{R}-10,97-98$ & 593.07 & Siderite & 2.8 & 3.2 & 25.1 & 70.2 & 891 & 145 & 144 & 0.2 & 0.1 & 0.1 & 0.2 & 127 & 15.2 & 24.9 & 6.0 \\
\hline $13 \mathrm{R}-3,11-13$ & 701.32 & Calcite & 17.3 & 0.8 & 0.2 & 46.3 & 53 & 522 & 237 & 0.5 & 0.1 & 0.1 & 0.4 & 14 & 23.0 & 70.4 & 0.9 \\
\hline $13 R-3,12-16$ & 701.33 & Calcite & 12.4 & 0.6 & 0.7 & 34.5 & 80 & 403 & 183 & 0.7 & 0.1 & 0.1 & 0.3 & 15 & 29.7 & 5.7 & 1.0 \\
\hline $15 \mathrm{R}-2,95-97$ & 719.05 & Siderite & 1.0 & 0.7 & 7.1 & 19.5 & 274 & 77 & 96 & 0.8 & 0.1 & 0.1 & 0.1 & 51 & 45.7 & 31.4 & 1.9 \\
\hline $15 \mathrm{R}-2,114-116$ & 719.34 & Siderite & 1.6 & 1.7 & 17.0 & 45.1 & 497 & 103 & 138 & 0.5 & 0.1 & 0.2 & 0.2 & 112 & 26.6 & 10.9 & 5.4 \\
\hline $16 \mathrm{R}-1,129-131$ & 728.29 & Siderite & 1.8 & 2.5 & 22.9 & 60.4 & 1340 & 145 & 175 & 0.4 & 0.1 & 0.1 & 0.2 & 182 & 17.6 & 13.2 & 4.8 \\
\hline $17 \mathrm{R}-3,147-149$ & 741.27 & Siderite & 1.7 & 2.3 & 24.6 & 63.1 & 765 & 133 & 176 & 0.3 & 0.1 & 0.1 & 0.2 & 133 & 9.9 & 10.1 & 4.8 \\
\hline $17 \mathrm{R}-4,97-99$ & 742.27 & Siderite & 2.1 & 2.8 & 27.3 & 71.5 & 810 & 152 & 206 & 0.3 & 0.1 & 0.1 & 0.3 & 158 & 11.0 & 28.3 & 7.0 \\
\hline $18 \mathrm{R}-3,116-119$ & 749.79 & Siderite & 1.2 & 1.9 & 21.1 & 53.4 & 539 & 106 & 121 & 0.4 & 0.1 & 0.1 & 0.2 & 112 & 24.4 & 13.5 & 4.3 \\
\hline $19 \mathrm{R}-1,11-13$ & 756.31 & Siderite & 1.6 & 3.7 & 19.2 & 56.6 & 609 & 125 & 172 & 0.5 & 0.1 & 0.1 & 0.1 & 131 & 19.6 & 2.8 & 3.8 \\
\hline $29 \mathrm{R}-6,27-29$ (treated) & 860.17 & Dolomite & 19.6 & 8.3 & 3.5 & 85.0 & 322 & 293 & 284 & 0.0 & 0.1 & 0.1 & 0.0 & 20 & 8.5 & 11.5 & 1.5 \\
\hline $33 \mathrm{R}-6,103-106$ & 899.63 & Calcite & 1.3 & 0.1 & 0.1 & $\begin{array}{l}3.0 \\
3.7\end{array}$ & $<2$ & 85.7 & 52 & 0.4 & 0.2 & 0.2 & 0.1 & 8 & 24.8 & 5.4 & $<0.5$ \\
\hline $37 \mathrm{R}-3,70-72$ (treated) & 933.40 & Dolomite & 20.0 & 9.3 & 0.8 & 83.6 & 135 & 313 & 230 & 0.1 & 0.0 & 0.1 & 0.0 & $\begin{array}{l}\circ \\
4\end{array}$ & 10.0 & 3.4 & $<0.5$ \\
\hline $41 \mathrm{R}-5,80-83$ & 974.28 & Calcite & 2.8 & 0.1 & 0.2 & $\begin{array}{r}5.0 \\
7.6\end{array}$ & 2 & 170 & 116 & 0.7 & 0.3 & 0.2 & 0.1 & 5 & 39.5 & 3.8 & $<0.5$ \\
\hline $44 \mathrm{R}-1,124-128$ (treated) & 997.74 & Dolomite & 16.0 & 5.9 & 1.7 & 63.8 & 258 & 333 & 152 & 0.2 & 0.1 & 0.1 & 0.1 & 10 & 30.0 & 2.8 & 1.0 \\
\hline $44 \mathrm{R}-5,131-133$ (treated) & 997.81 & Dolomite & 19.1 & 7.1 & 2.2 & 77.0 & 2 & 303 & 159 & 0.0 & 0.1 & 0.1 & 0.0 & 13 & 18.9 & 7.3 & 1.3 \\
\hline $45 \mathrm{R}-1,73-75$ (treated) & 1006.93 & Dolomite & 18.0 & 7.7 & 1.6 & 74.9 & 122 & 276 & 165 & 0.0 & 0.1 & 0.1 & 0.1 & 7 & 21.5 & 5.8 & 0.9 \\
\hline $51 \mathrm{R}-7,125-127$ & 1063.37 & Calcite & 1.3 & 0.1 & 0.2 & 3.8 & $<2$ & 108 & 78 & 0.5 & 0.3 & 0.2 & 0.1 & 3 & 53.9 & 2.6 & $<0.5$ \\
\hline $52 \mathrm{R}-1,9-12$ & 1064.19 & Dolomite & 19.2 & 7.1 & 4.9 & 82.5 & 297 & 305 & 173 & 0.0 & 0.1 & 0.1 & 0.1 & 12 & 10.6 & 3.2 & 1.7 \\
\hline $52 \mathrm{R}-1,93-96$ & 1065.03 & Calcite & 14.5 & 0.8 & 0.5 & 40.0 & 57 & 754 & 123 & 0.5 & 0.1 & 0.2 & 0.0 & 6 & 44.4 & 3.8 & $<0.5$ \\
\hline $56 \mathrm{R}-6,67-70$ & 1109.81 & Calcite & 15.2 & 0.1 & 0.2 & 38.6 & 48 & 736 & 108 & 0.5 & 0.1 & 0.1 & 0.3 & 2 & 20.9 & 9.9 & $<0.5$ \\
\hline \multicolumn{18}{|l|}{$150-903 \mathrm{D}-$} \\
\hline $18 R-1,66-71$ & 939.06 & Dolomite & 16.6 & 8.1 & 0.8 & 71.1 & 151 & 276 & 210 & 0.1 & 0.0 & 0.1 & 0.0 & 6 & 13.1 & 5.9 & $<0.5$ \\
\hline $21 \mathrm{R}-8,46-49$ & 1005.92 & Dolomite & 18.2 & 7.3 & 1.2 & 73.1 & 138 & 367 & 176 & 0.1 & 0.0 & 0.1 & 0.0 & 13 & 17.4 & 9.3 & 0.8 \\
\hline \multicolumn{18}{|l|}{$\begin{array}{l}150-905 \mathrm{~A}- \\
64 \mathrm{X}-5,65-68 \text { (treated) }\end{array}$} \\
\hline \multirow{2}{*}{\multicolumn{18}{|c|}{$150-906 \mathrm{~A}$}} \\
\hline & & & & & & & & & & & & & 0.1 & 15 & 34.2 & 15.0 & 0.9 \\
\hline $8 \mathrm{X}-3,53-55$ & 66.03 & Siderite & 1.1 & 2.0 & 16.2 & 43.1 & 620 & 91.4 & 74 & 0.5 & 0.1 & 0.1 & 0.1 & 83 & 21.8 & 9.3 & 3.4 \\
\hline $10 X-7,21-24$ & 90.61 & Dolomite & 13.5 & 6.5 & 1.2 & 58.8 & 109 & 664 & 155 & 0.3 & 0.1 & 0.2 & 0.2 & 23 & 23.8 & 28.2 & 1.0 \\
\hline $11 X-5,55-58$ & 98.05 & Siderite & 1.4 & 2.3 & 20.3 & 53.3 & 706 & 108 & 116 & 0.5 & 0.1 & 0.1 & 0.2 & 111 & 21.3 & 12.3 & 4.0 \\
\hline $29 \times-6,17-20$ & 272.77 & Siderite & 0.3 & 0.4 & 3.7 & 9.8 & 145 & 42.1 & 43 & 0.7 & 0.1 & 0.1 & 0.1 & 26 & 61.2 & 3.5 & 1.1 \\
\hline $36 \mathrm{X}-2,145-147$ & 336.15 & Dolomite & 8.0 & 4.3 & 0.7 & 36.0 & 281 & 150 & 8 & 0.1 & $<.01$ & 0.0 & 0.1 & 5 & 5.6 & 9.2 & $<0.5$ \\
\hline $42 X-2,6-13$ & 392.33 & Dolomite & $\begin{array}{l}0.0 \\
9.3\end{array}$ & 4.8 & 0.1 & 40.3 & $\begin{array}{r}201 \\
1750\end{array}$ & 214 & $210^{\circ}$ & 0.1 & 0.0 & 0.1 & 0.1 & 20 & $\begin{array}{r}13.0 \\
13.4\end{array}$ & 26.2 & $<0.5$ \\
\hline $54 X-5,49-53$ & 512.69 & Siderite & 1.4 & 1.9 & 11.7 & 34.2 & 722 & 58.9 & 73 & 0.2 & 0.1 & 0.1 & 0.0 & 25 & 34.3 & 1.1 & 2.1 \\
\hline
\end{tabular}

Notes: Carbonate $=\mathrm{wt} \%$ of the bulk sample, assuming all $\mathrm{Ca}, \mathrm{Mg}$, and $\mathrm{Fe}$ are derived from carbonate; treated $=\mathrm{NaOAc}$ removal of calcite; $*=$ separate sample from $89-94-\mathrm{cm}$ interval. 
Table 2. Calculated elemental composition of the diagenetic carbonates from Leg 150.

\begin{tabular}{|c|c|c|c|c|c|}
\hline $\begin{array}{l}\text { Core, section, } \\
\text { interval }(\mathrm{cm})\end{array}$ & $\begin{array}{l}\text { Depth } \\
\text { (mbsf) }\end{array}$ & Mineralogy & $\begin{array}{c}\mathrm{Ca} \\
(\mathrm{mol} \%)\end{array}$ & $\begin{array}{c}\mathrm{Mg} \\
(\mathrm{mol} \%)\end{array}$ & $\begin{array}{c}\mathrm{Fe} \\
(\mathrm{mol} \%)\end{array}$ \\
\hline $\begin{array}{l}\text { 150-902D- } \\
21 X-3,121-126 \\
30 X-6,13-17 \\
48 X-3,7-9 \\
48 X-4,102-104 \\
48 X-6,16-18 \\
51 X-3,65-67 \\
63 X-1,1-5\end{array}$ & $\begin{array}{l}174.24 \\
265.33 \\
433.67 \\
436.12 \\
438.26 \\
462.55 \\
574.41\end{array}$ & $\begin{array}{l}\text { Calcite } \\
\text { Dolomite } \\
\text { Siderite/francolite } \\
\text { Siderite } \\
\text { Siderite } \\
\text { Dolomite } \\
\text { Siderite }\end{array}$ & $\begin{array}{r}91.7 \\
52.1 \\
29.6 \\
7.3 \\
6.5 \\
50.9 \\
6.7\end{array}$ & $\begin{array}{r}7.4 \\
45.1 \\
16.8 \\
21.1 \\
18.1 \\
44.7 \\
13.9\end{array}$ & $\begin{array}{r}0.9 \\
2.8 \\
53.7 \\
71.7 \\
75.4 \\
4.4 \\
79.4\end{array}$ \\
\hline $\begin{array}{l}150-903 \mathrm{~A}- \\
11 \mathrm{H}-3,145-150 \text { (untreated) } \\
24 \mathrm{X}-3,145-150 \text { (untreated) } \\
41 \mathrm{X}-1,89-94 \\
41 \mathrm{X}-1,89-94^{*} \\
44 \mathrm{X}-5,103-109 \\
45 \mathrm{X}-2,12-19 \text { (untreated) } \\
47 \mathrm{X}-2,56-62 \text { (untreated) } \\
51 \mathrm{X}-3,137-139 \\
63 \mathrm{X}-6,148-150 \\
64 \mathrm{X}-4,36-38 \text { (treated) } \\
69 \mathrm{X}-5,17-19 \\
70 \mathrm{X}-2,76-78 \\
71 \mathrm{X}-1,103-106 \text { (untreated) }\end{array}$ & $\begin{array}{r}99.45 \\
196.35 \\
356.59 \\
356.59 \\
391.60 \\
395.92 \\
415.76 \\
456.57 \\
576.78 \\
582.36 \\
631.77 \\
637.46 \\
648.71\end{array}$ & $\begin{array}{l}\text { Dolomite/calcite } \\
\text { Dolomite/calcite } \\
\text { Calcite } \\
\text { Calcite } \\
\text { Siderite } \\
\text { Calcite/dolomite } \\
\text { Calcite/siderite } \\
\text { Calcite } \\
\text { Siderite } \\
\text { Siderite } \\
\text { Siderite } \\
\text { Siderite } \\
\text { Calcite/siderite }\end{array}$ & $\begin{array}{r}59.6 \\
59.3 \\
90.2 \\
90.5 \\
19.7 \\
69.8 \\
75.8 \\
89.2 \\
8.1 \\
8.1 \\
8.0 \\
11.7 \\
76.8\end{array}$ & $\begin{array}{r}26.5 \\
27.4 \\
8.8 \\
8.7 \\
12.4 \\
22.0 \\
10.5 \\
9.6 \\
21.2 \\
31.7 \\
23.4 \\
30.2 \\
14.1\end{array}$ & $\begin{array}{r}14.0 \\
13.3 \\
1.0 \\
0.8 \\
67.9 \\
8.2 \\
13.7 \\
1.2 \\
70.8 \\
60.1 \\
68.6 \\
58.1 \\
9.1\end{array}$ \\
\hline $\begin{array}{l}\text { 150-903C- } \\
10 \mathrm{R}-10,97-98 \\
13 \mathrm{R}-3,11-13 \\
13 \mathrm{R}-3,12-16 \\
15 \mathrm{R}-2,95-97 \\
15 \mathrm{R}-2,114-116 \\
16 \mathrm{R}-1,129-131 \\
17 \mathrm{R}-3,147-149 \\
17 \mathrm{R}-4,97-99 \\
18 \mathrm{R}-3,116-1197 \\
19 \mathrm{R}-1,11-13 \\
29 \mathrm{R}-6,27-29 \text { (treated) } \\
33 \mathrm{R}-6,103-106 \\
37 \mathrm{R}-3,70-72 \text { (treated) } \\
41 \mathrm{R}-5,80-83 \\
44 \mathrm{R}-1,124-128 \text { (treated) } \\
44 \mathrm{R}-5,131-133 \text { (treated) } \\
45 \mathrm{R}-1,73-75 \text { (treated) } \\
\text { 51R-7, 125-127 } \\
\text { 52R-1,9-12 } \\
52 \mathrm{R}-1,93-96 \\
56 \mathrm{R}-6,67-70\end{array}$ & $\begin{array}{r}593.07 \\
701.32 \\
701.33 \\
719.05 \\
719.34 \\
728.29 \\
741.27 \\
742.27 \\
49.79 \\
756.31 \\
860.17 \\
899.63 \\
933.40 \\
974.28 \\
997.74 \\
997.81 \\
1006.93 \\
1063.37 \\
1064.19 \\
1065.03 \\
1109.81\end{array}$ & $\begin{array}{l}\text { Siderite } \\
\text { Calcite } \\
\text { Calcite } \\
\text { Siderite } \\
\text { Siderite } \\
\text { Siderite } \\
\text { Siderite } \\
\text { Siderite } \\
\text { Siderite } \\
\text { Siderite } \\
\text { Dolomite } \\
\text { Calcite } \\
\text { Dolomite } \\
\text { Calcite } \\
\text { Dolomite } \\
\text { Dolomite } \\
\text { Dolomite } \\
\text { Calcite } \\
\text { Dolomite } \\
\text { Calcite } \\
\text { Calcite }\end{array}$ & $\begin{array}{l}10.7 \\
92.4 \\
89.2 \\
13.2 \\
9.71 \\
7.9 \\
7.3 \\
8.0 \\
6.3 \\
7.3 \\
54.7 \\
88.4 \\
55.9 \\
90.5 \\
59.5 \\
58.9 \\
56.6 \\
84.1 \\
55.9 \\
89.9 \\
98.2\end{array}$ & $\begin{array}{r}20.2 \\
6.7 \\
7.2 \\
16.3 \\
6.7 \\
6.7 \\
18.2 \\
16.3 \\
17.4 \\
16.2 \\
28.5 \\
38.2 \\
6.7 \\
42.6 \\
6.0 \\
36.0 \\
36.2 \\
39.8 \\
8.7 \\
33.9 \\
7.8 \\
1.1\end{array}$ & $\begin{array}{c}69.0 \\
0.9 \\
3.6 \\
70.6 \\
73.6 \\
73.9 \\
76.4 \\
74.5 \\
77.6 \\
64.2 \\
7.1 \\
4.9 \\
1.5 \\
3.5 \\
4.5 \\
4.94 \\
3.6 \\
7.1 \\
10.2 \\
2.4 \\
0.7\end{array}$ \\
\hline $\begin{array}{l}150-903 \mathrm{D}- \\
18 \mathrm{R}-1,66-71 \\
21 \mathrm{R}-8,46-49\end{array}$ & $\begin{array}{r}939.06 \\
1005.92\end{array}$ & $\begin{array}{l}\text { Dolomite } \\
\text { Dolomite }\end{array}$ & $\begin{array}{l}54.4 \\
58.6\end{array}$ & $\begin{array}{l}43.8 \\
38.7\end{array}$ & $\begin{array}{l}1.9 \\
2.7\end{array}$ \\
\hline $\begin{array}{l}150-905 \mathrm{~A}- \\
64 \mathrm{X}-5,65-68\end{array}$ & 574.90 & Dolomite & 53.2 & 45.0 & 1.8 \\
\hline $\begin{array}{l}150-906 \mathrm{~A}- \\
5 \mathrm{H}-3,145-150 \text { (untreated) } \\
8 \mathrm{X}-3,53-55 \\
10 \mathrm{X}-7,21-24 \\
11 \mathrm{X}-5,55-58 \\
29 \mathrm{X}-6,17-20 \\
36 \mathrm{X}-2,145-147 \\
42 \mathrm{X}-2,6-13 \\
54 \mathrm{X}-5,49-53 \\
\end{array}$ & $\begin{array}{r}41.97 \\
66.03 \\
90.61 \\
98.05 \\
272.77 \\
336.15 \\
392.33 \\
512.69 \\
\end{array}$ & $\begin{array}{l}\text { Dolomite/calcite } \\
\text { Siderite } \\
\text { Dolomite } \\
\text { Siderite } \\
\text { Siderite } \\
\text { Dolomite } \\
\text { Dolomite } \\
\text { Siderite }\end{array}$ & $\begin{array}{r}68.6 \\
6.9 \\
53.7 \\
6.9 \\
7.5 \\
51.4 \\
53.6 \\
10.6\end{array}$ & $\begin{array}{l}18.2 \\
20.2 \\
42.8 \\
18.9 \\
19.6 \\
45.6 \\
45.8 \\
24.1 \\
\end{array}$ & $\begin{array}{r}13.2 \\
72.9 \\
3.4 \\
74.2 \\
73.0 \\
3.1 \\
0.6 \\
65.3 \\
\end{array}$ \\
\hline
\end{tabular}

Notes: treated $=\mathrm{NaOAc}$ removal of calcite; ${ }^{*}=$ separate sample from $89-94-\mathrm{cm}$ interval.

The pore-water $\mathrm{Ca}$ concentration decreases rapidly to $3.6 \mathrm{mM}$ by 14 mbsf, remains fairly constant to a depth of $225 \mathrm{mbsf}$, and then steadily increases with depth (Fig. 9). An initial decrease in Ca, associated with an increase in alkalinity from sulfate reduction, is commonly observed in rapidly deposited, organic-rich sediments and is generally related to carbonate precipitation (Gieskes, 1981). The pore-water $\mathrm{Mg}$ ion concentration decreases to a minimum value of $27.8 \mathrm{mM}$ at $176 \mathrm{mbsf}$. The decrease in pore-water $\mathrm{Ca}$ and $\mathrm{Mg}$ on the New Jersey Margin may result from the direct precipitation of dolomite,

$$
\mathrm{Ca}^{2+}+\mathrm{Mg}^{2+}+2 \mathrm{HCO}_{3}^{-}=\mathrm{CaMg}\left(\mathrm{CO}_{3}\right)_{2}+2 \mathrm{H}^{+}
$$

The pore-water $\mathrm{Mg} / \mathrm{Ca}$ ratio increases sharply in the uppermost 100 mbsf at the upper slope sites to maximum values of 11 to 18 . The increased $\mathrm{Mg} / \mathrm{Ca}$ ratio favors dolomitization of calcite,

$$
\mathrm{Mg}^{2+}+2 \mathrm{CaCO}_{3}=\mathrm{CaMg}\left(\mathrm{CO}_{3}\right)_{2}+\mathrm{Ca}^{2+}
$$

The $\mathrm{Mg} / \mathrm{Ca}$ pore-water ratio decreases asymptotically to a value of approximately 1 , a trend that is commonly observed in marine sediments where dolomite is precipitating. $\mathrm{A} \mathrm{Mg} / \mathrm{Ca}$ ratio equal to one is predicted for pore water in thermodynamic equilibrium with dolomite (Robbie et al., 1978; Compton, 1988b).

The range in $\delta^{13} \mathrm{C}$ values ( $-15.0 \%$ to $+4.5 \%$ PDB) of dolomite from Leg 150 indicates that the carbon was primarily derived from the dissolution of biogenic calcite and degradation of organic matter throughout the sulfate-reduction zone and in the upper methanogenesis zone. The lack of large positive $\delta^{13} \mathrm{C}$ values for the dolomite samples suggests that most of the dolomite did not form in the deeper portions of the methanogenesis zone. Replacement of biogenic calcite by dolomite is supported by SEM photomicrographs (Pl. 1), foraminiferal ghosts, and nannofossils partially replaced by rhombohedral 


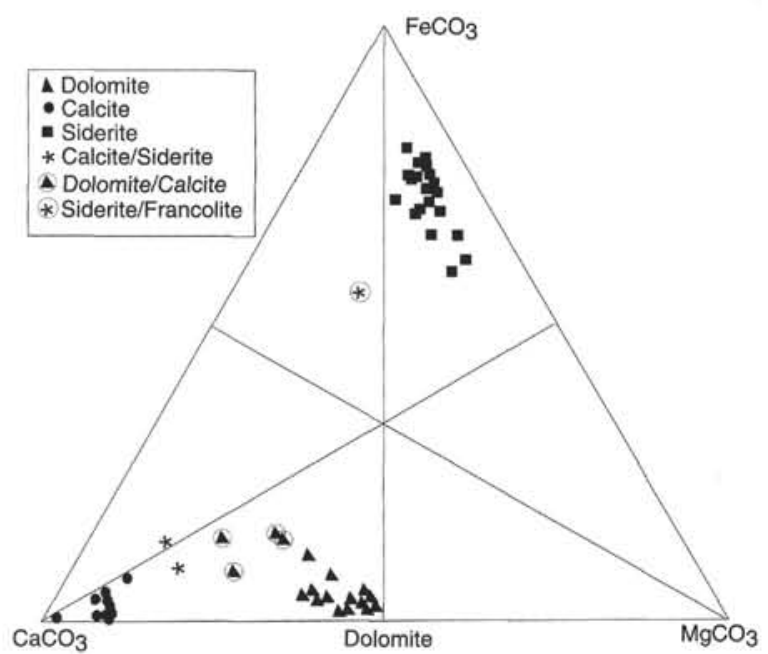

Figure 3. Ternary diagram depicting the compositional fields of calcite, dolomite, and siderite. There are a total of 52 samples consisting of 11 calcite, 14 dolomite, 20 siderite, 4 dolomite/calcite, 2 calcite/siderite, and 1 siderite/francolite.

carbonates. The general lack of calcareous fossils in the middle and upper Miocene sediments of these upper slope sites (Mountain, Miller, Blum, et al., 1994) reflects their transformation to diagenetic carbonates. In fact, diagenetic carbonate formation was probably limited in these sediments by the availability of precursor biogenic carbonate. The $\delta^{18} \mathrm{O}$ values of the dolomite decrease with burial depth, indicating that either dolomite formed at those depths or at shallow depths (lower temperatures) and then recrystallized upon deeper burial (Fig. 6). The similar $\delta^{18} \mathrm{O}$ values of Site 906 dolomites to more deeply buried Site 903 dolomites can be explained by rapid burial at Site 903 since the Pleistocene with $350 \mathrm{~m}$ of Pleistocene sediment at Site 903 compared to $50 \mathrm{~m}$ at Site 906 (Fig. 2). The dolomite $\delta^{18} \mathrm{O}$ values of Site 903 have apparently not reequilibrated since their rapid burial in the Pleistocene. The temperature of formation of dolomite is estimated to range from $8^{\circ} \mathrm{C}$ to $30^{\circ} \mathrm{C}$ using the proto-dolomite-water fractionation expression of Fritz and Smith (1970). This temperature range is consistent with the present-day bottom-water temperatures and a geothermal gradient of approximately $30^{\circ}$ to $35^{\circ} \mathrm{C} / \mathrm{km}$. The unusually low $\delta^{18} \mathrm{O}$ values of $-7.8 \%$ to $-5.5 \%$ of mixed dolomite/calcite samples in the uppermost 200 mbsf yield formation temperatures of 55 to $70^{\circ} \mathrm{C}$. The same low $\delta^{18} \mathrm{O}$ values were obtained for untreated (dolomite and calcite) and treated (dolomite only) samples (Table 3). Such high temperatures are inconsistent with the shallow burial depths of these carbonates, which are currently forming at depth in the sediment. Although negative $\delta^{18} \mathrm{O}$ values can reflect a meteoric water influence, a meteoric influence is not supported by the porewater chemistry. Therefore, these low $\delta^{18} \mathrm{O}$ values are enigmatic.

Some carbonate cemented samples contain a mixture of dolomite and calcite (Fig. 3). The low iron content of the calcite samples (maximum of $7 \mathrm{~mol} \% \mathrm{Fe}$ ) indicates that the dolomite/calcite samples either contain dolomite with $\mathrm{Fe}$ contents of around $20 \mathrm{~mol} \% \mathrm{Fe}$, significantly higher than the maximum $\mathrm{Fe}$ content of the dolomite field (10 $\mathrm{mol} \% \mathrm{Fe}$ ), or that approximately $5 \%$ of the carbonate in these samples is siderite. Similarly, the $\mathrm{Mg}$ content $(14.1 \mathrm{~mol} \% \mathrm{Mg})$ of the calcite/siderite Sample 150-903A-71X-1, 103-106 cm, requires that either the siderite has a $40 \mathrm{~mol} \% \mathrm{Mg}$ content, higher than the maximum of $30 \mathrm{~mol} \% \mathrm{Mg}$ in the defined siderite field, or that approximately $5 \%$ of the carbonate fraction of this sample is dolomite. It is difficult to determine unequivocally if these mixed samples contain minor amounts of siderite or dolomite, because the siderite and dolomite contents of the bulk samples are near the detection limit of the XRD
(0.5 wt $\%)$. An equilibrium mixture of calcite, ferroan dolomite, and siderite in these samples would indicate an expansion of the threephase field at low sedimentary temperatures, consistent with expansion of the three-phase field between $550^{\circ}$ and $250^{\circ} \mathrm{C}$ in the thermodynamically calculated phase diagrams of Anovitz and Essene (1987).

\section{Siderite}

Siderite precipitation on the New Jersey Margin is enhanced by (1) an increase in the degree of supersaturation attributed to an increase in carbonate alkalinity, (2) high $\mathrm{Fe}$ pore-water concentrations (Fig. 10), and (3) low sulfate concentrations (Mozley and Burns, 1993). Of these factors, the Fe content of the pore water is the most important in promoting the precipitation of siderite. The source of Fe for siderite is most likely the reduction of $\mathrm{Fe}$ oxyhydroxide coatings on the abundant terrigenous material at these sites. At Site 903, where the sulfate ion is largely depleted to near zero by $14 \mathrm{mbsf}$, pore-water Fe concentrations initially increase to a maximum of $54 \mu \mathrm{M}$ at 50 mbsf, then decrease and maintain an average concentration of $8 \mu \mathrm{M}$ between 110 and $540 \mathrm{mbsf}$. Another maximum of $72 \mu \mathrm{M}$ at $588 \mathrm{mbsf}$ is associated with the siderite-rich interval from 550 to $775 \mathrm{mbsf}$ (Fig. 10). Although similarly high Fe concentrations were confirmed, the pore-water Fe profile at Site 903 below 500 mbsf was not reproduced in shore-based analyses using DCP. Siderite appears to be forming currently in the uppermost $100 \mathrm{mbsf}$ within the methanogenesis zone at Site 906 (Fig. 2). At Site 906, sulfate ion concentrations decrease to near zero and pore-water $\mathrm{Fe}$ concentrations reach a local maximum of $11 \mu \mathrm{M}$ by $32 \mathrm{mbsf}$ (Mountain, Miller, Blum, et al., 1994). Substitution of $\mathrm{Mg}$ is favored over $\mathrm{Ca}$ in siderite because the ionic radius of $\mathrm{Mg}$ is more similar to that of $\mathrm{Fe}$, and $\mathrm{Mg}$ is more abundant than $\mathrm{Ca}$ in these pore waters (Fig. 9). The pore-water Ca concentration is fairly constant, increasing only slightly in the methanogenesis zone, whereas the pore-water $\mathrm{Mg} / \mathrm{Fe}$ ratio is more variable and probably contributed to the large variation in $\mathrm{Mg}$ content of the siderite samples (Fig. 3).

Mozley and Carothers (1992) found that siderite concretions which formed early in association with glaucony-rich intervals have higher $\mathrm{Fe} / \mathrm{Mg}$ ratios than those that formed at greater burial depths in the methanogenesis zone. In contrast to the results of Mozley and Carothers (1992), New Jersey Margin siderites associated with glaucony-rich intervals have low $\mathrm{Fe} / \mathrm{Mg}$ ratios which increase with increasing stratigraphic distance from the glaucony-rich intervals. Siderites that precipitate concurrently with glaucony during suboxic diagenesis are expected to have lower $\mathrm{Fe} / \mathrm{Mg}$ ratios, because glaucony formation should remove $\mathrm{Fe}$ and lower the pore-water $\mathrm{Fe} / \mathrm{Mg}$ ratio.

Consistently positive $\delta^{13} \mathrm{C}$ values indicate siderite precipitation in the methanogenesis zone rather than in either the suboxic zone of microbial iron reduction associated with glaucony formation (McCracken et al., this volume) or the sulfate-reduction zone (Fig. 11). Siderite precipitation is possible in the suboxic zone of microbial iron reduction because $\mathrm{Fe}^{2+}$ is released. Iron removal in the suboxic zone by sulfide minerals is limited. Suboxic conditions are necessary for glaucony formation due to the presence of both $\mathrm{Fe}^{2+}$ and $\mathrm{Fe}^{3+}$ in the mineral structure (Ireland et al., 1983; Odin and Matter, 1981). Siderite that precipitates in the suboxic zone with glaucony should have depleted $\delta^{13} \mathrm{C}$ values compared to siderites that precipitate in the methanogenesis zone (Mozley and Carothers, 1992). Therefore, although Leg 150 siderites associated with glaucony-rich intervals have low $\mathrm{Fe} / \mathrm{Mg}$ ratios, they probably did not form concurrently with the glaucony in the suboxic zone but rather formed later in the deeper methanogenesis zone. The positive $\delta^{13} \mathrm{C}$ values of the siderite are consistent with the high sedimentation rates of Fe-rich terrigenous sediment and organic matter on the upper slope of the New Jersey Margin (Mozley and Burns, 1993). Dolomite is favored over siderite in the sulfatereduction zone because the formation of monosulfides, and eventual- 

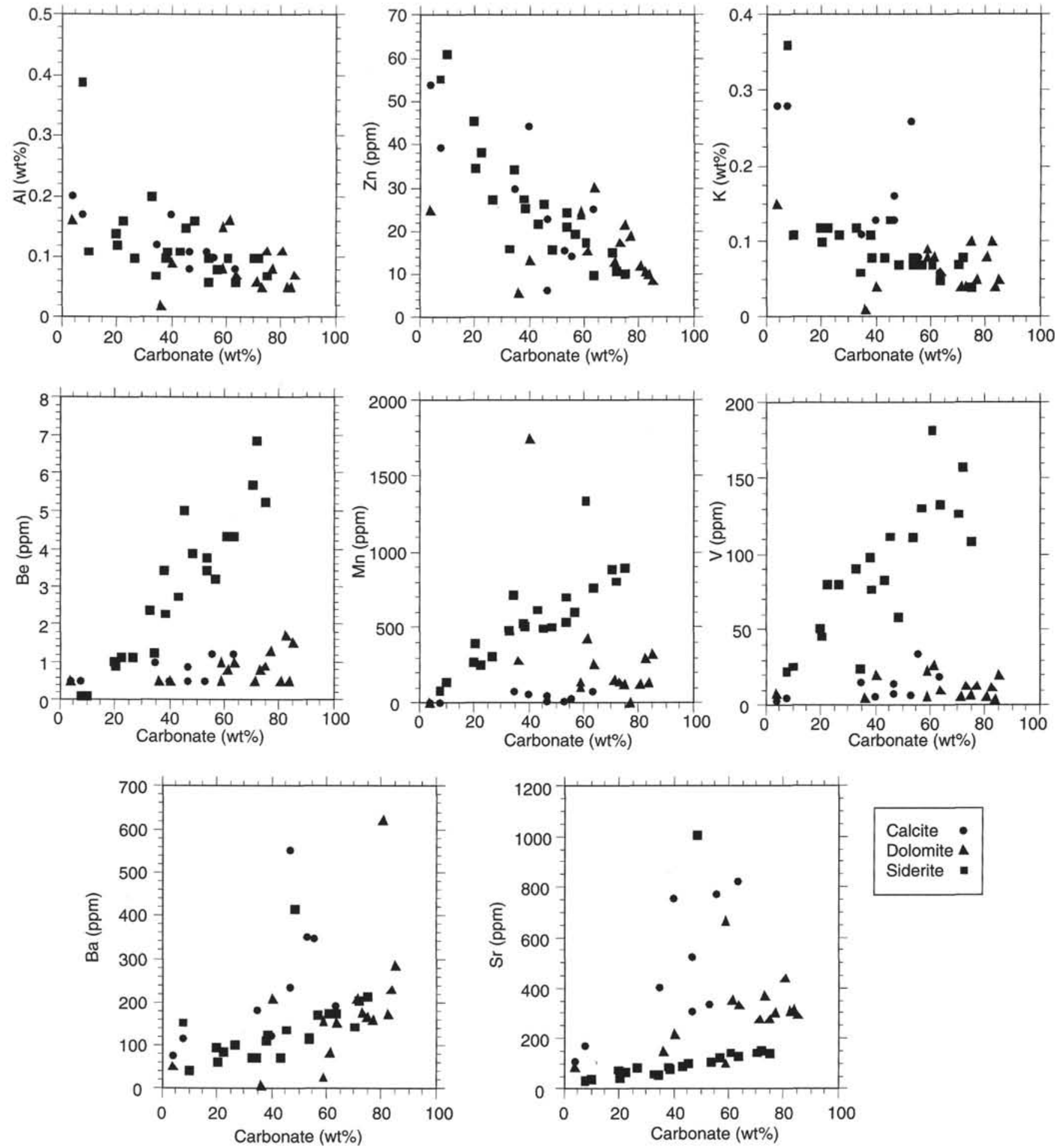

Figure 4. Concentrations of $\mathrm{Al}, \mathrm{Zn}, \mathrm{K}, \mathrm{Be}, \mathrm{Mn}, \mathrm{V}, \mathrm{Ba}$, and $\mathrm{Sr}$ in the acid-soluble fraction of the bulk samples as a function of weight percent carbonate. The siderite sample with the highest $\mathrm{Sr}$ content is a mixture of siderite and francolite.

ly pyrite, rapidly removes $\mathrm{Fe}^{2+}$ from the pore water (Berner, 1984). Siderite precipitation can occur in the sulfate reduction zone only if the reduction of iron exceeds the rate of sulfate-reduction (Pye et al., 1990).

The trend of decreasing siderite $\delta^{18} \mathrm{O}$ values with depth at Sites 903 and 906 probably reflects siderite formation or recrystallization at the higher temperatures of deeper burial (Fig. 6). The similar $\delta^{18} \mathrm{O}$ values of Site 906 siderites with the more deeply buried Site 903 siderites can be explained by the rapid burial at Site 903 since the Pleistocene ( $350 \mathrm{~m}$ of Pleistocene sediment at Site 903 compared to $50 \mathrm{~m}$ of Pleistocene at Site 906 , Fig. 2). $\delta^{18} \mathrm{O}$ values of siderites from this study are compatible with precipitation at shallow burial depths in a temperature range of $15^{\circ}-30^{\circ} \mathrm{C}$. $\delta^{18} \mathrm{O}$ values of most Paleozoic and Mesozoic siderite concretionary cements are lower than expected for 
Table 3. Mineralogy and isotopic composition of diagenetic carbonate samples from Leg 150.

\begin{tabular}{|c|c|c|c|c|c|}
\hline $\begin{array}{l}\text { Core, section, } \\
\text { interval }(\mathrm{cm})\end{array}$ & $\begin{array}{l}\text { Depth } \\
\text { (mbsf) }\end{array}$ & Mineralogy & $\begin{array}{c}\delta^{13} \mathrm{C} \\
(\% \circ \mathrm{PDB})\end{array}$ & $\begin{array}{c}\delta^{18} \mathrm{O} \\
\text { (\%。 PDB) } \\
\text { (uncorr.) }\end{array}$ & $\begin{array}{c}\delta^{18} \mathrm{O} \\
\text { (\% PDB) } \\
\text { (corr.) }\end{array}$ \\
\hline $\begin{array}{l}\text { 150-902D- } \\
21 X-3,121-126 \\
30 X-6,13-17 \\
48 X-3,7-9 \\
48 X-4,102-104 \\
51 X-3,65-67 \\
63 X-1,1-5\end{array}$ & $\begin{array}{l}174.24 \\
265.33 \\
433.67 \\
436.12 \\
462.55 \\
574.41\end{array}$ & $\begin{array}{l}\text { Calcite } \\
\text { Dolomite } \\
\text { Siderite/francolite } \\
\text { Siderite } \\
\text { Dolomite } \\
\text { Siderite }\end{array}$ & $\begin{array}{r}-22.1 \\
-15.0 \\
2.2 \\
2.9 \\
-8.8 \\
-1.1\end{array}$ & $\begin{array}{l}1.9 \\
3.8 \\
2.2 \\
2.9 \\
2.7 \\
4.5\end{array}$ & $\begin{array}{l}1.9 \\
3.0 \\
0.7 \\
1.4 \\
1.9 \\
3.1\end{array}$ \\
\hline $\begin{array}{l}150-903 \mathrm{~A}- \\
11 \mathrm{H}-3,145-150 \text { (treated) } \\
11 \mathrm{H}-3,145-150 \text { (untreated) } \\
24 \mathrm{X}-3,145-150 \text { (treated) } \\
24 \mathrm{X}-3,145-150 \text { (untreated) } \\
41 \mathrm{X}-1,89-94 \\
41 \mathrm{X}-1,89-94^{\mathrm{a}} \\
48 \mathrm{X}-5,20-22^{\mathrm{a}} \\
48 \mathrm{X}-5,95-97^{\mathrm{a}} \\
51 \mathrm{X}-3,137-139 \\
51 \mathrm{X}-3,137-139^{\mathrm{a}} \\
63 \mathrm{X}-6,148-150 \\
64 \mathrm{X}-4,136-138^{\mathrm{a}} \\
69 \mathrm{X}-5,17-19 \\
70 \mathrm{X}-2,76-78 \\
13 \mathrm{R}-3,12-16 \\
15 \mathrm{R}-2,95-97\end{array}$ & $\begin{array}{r}99.45 \\
99.45 \\
196.35 \\
196.35 \\
356.59 \\
356.59 \\
428.51 \\
429.25 \\
456.36 \\
456.36 \\
576.78 \\
582.86 \\
631.77 \\
637.46 \\
701.33 \\
719.05\end{array}$ & $\begin{array}{l}\text { Dolomite } \\
\text { Dolomite/calcite } \\
\text { Dolomite } \\
\text { Dolomite/calcite } \\
\text { Calcite } \\
\text { Calcite } \\
\text { Calcite } \\
\text { Calcite/siderite } \\
\text { Calcite } \\
\text { Calcite } \\
\text { Siderite } \\
\text { Calcite } \\
\text { Siderite } \\
\text { Siderite } \\
\text { Calcite } \\
\text { Siderite }\end{array}$ & $\begin{array}{r}-1.3 \\
-1.5 \\
0.3 \\
0.3 \\
-22.9 \\
-21.6 \\
-16.7 \\
-18.7 \\
-19.5 \\
-18.5 \\
5.9 \\
-0.9 \\
5.4 \\
8.8 \\
-37.3 \\
7.4\end{array}$ & $\begin{array}{r}-7.0 \\
-7.3 \\
-4.7 \\
-4.6 \\
3.2 \\
4.3 \\
4.0 \\
4.3 \\
2.6 \\
3.8 \\
5.0 \\
5.4 \\
5.4 \\
5.0 \\
0.8 \\
3.6\end{array}$ & $\begin{array}{r}-7.8 \\
-8.1 \\
-5.5 \\
-5.4 \\
3.2 \\
4.3 \\
4.0 \\
2.9 \\
2.6 \\
3.8 \\
3.6 \\
5.4 \\
4.0 \\
3.6 \\
0.8 \\
2.2\end{array}$ \\
\hline $\begin{array}{l}150-903 \mathrm{C}- \\
10 \mathrm{R}-10,97-98^{\mathrm{a}} \\
15 \mathrm{R}-2,114-116^{\mathrm{a}} \\
16 \mathrm{R}-1,129-131 \\
17 \mathrm{R}-3,147-149 \\
18 \mathrm{R}-3,116-119 \\
19 \mathrm{R}-1,11-13 \\
29 \mathrm{R}-6,27-29 \text { (treated) } \\
29 \mathrm{R}-6,27-29^{\mathrm{a}} \\
37 \mathrm{R}-3,70-72 \\
37 \mathrm{R}-3,70-72^{\mathrm{a}} \\
41 \mathrm{R}-5,80-83^{\mathrm{a}} \\
44 \mathrm{R}-5,131-133 \text { (treated) } \\
51 \mathrm{R}-6,145-147^{\mathrm{a}} \\
51 \mathrm{R}-7,125-127 \\
51 \mathrm{R}-7,125-127^{\mathrm{a}} \\
52 \mathrm{R}-1,9-12^{\mathrm{a}} \\
\text { 56R-6, 67-70 }\end{array}$ & $\begin{array}{r}593.07 \\
719.34 \\
728.29 \\
741.29 \\
749.79 \\
756.31 \\
860.17 \\
860.17 \\
933.40 \\
933.40 \\
974.30 \\
997.81 \\
1062.07 \\
1063.37 \\
1063.37 \\
1064.19 \\
1109.81\end{array}$ & $\begin{array}{l}\text { Siderite } \\
\text { Siderite } \\
\text { Siderite } \\
\text { Siderite } \\
\text { Siderite } \\
\text { Siderite } \\
\text { Dolomite } \\
\text { Dolomite } \\
\text { Dolomite } \\
\text { Dolomite } \\
\text { Calcite } \\
\text { Dolomite } \\
\text { Calcite } \\
\text { Calcite } \\
\text { Calcite } \\
\text { Dolomite } \\
\text { Calcite }\end{array}$ & $\begin{array}{r}2.7 \\
7.3 \\
4.5 \\
2.3 \\
3.1 \\
4.2 \\
-0.1 \\
-1.8 \\
-4.8 \\
-4.4 \\
0.6 \\
-0.5 \\
-0.8 \\
-1.8 \\
-2.8 \\
-1.8 \\
0.4\end{array}$ & $\begin{array}{r}6.6 \\
5.4 \\
3.5 \\
3.8 \\
4.5 \\
4.5 \\
0.2 \\
2.1 \\
0.5 \\
1.8 \\
-0.4 \\
-0.9 \\
-0.1 \\
-0.3 \\
-0.4 \\
-0.9 \\
-1.4\end{array}$ & $\begin{array}{r}5.2 \\
4.0 \\
2.1 \\
2.4 \\
3.1 \\
3.1 \\
-0.6 \\
1.3 \\
-0.3 \\
1.0 \\
-0.4 \\
-1.7 \\
-0.1 \\
-0.3 \\
-0.4 \\
-1.7 \\
-1.4\end{array}$ \\
\hline $\begin{array}{l}150-903 \mathrm{D}- \\
18 \mathrm{R}-1,66-71 \\
21 \mathrm{R}-8,46-49\end{array}$ & $\begin{array}{r}939.06 \\
1005.92\end{array}$ & $\begin{array}{l}\text { Dolomite } \\
\text { Dolomite }\end{array}$ & $\begin{array}{l}-3.5 \\
-2.2\end{array}$ & $\begin{array}{r}0.5 \\
-0.6\end{array}$ & $\begin{array}{l}-0.3 \\
-1.4\end{array}$ \\
\hline $\begin{array}{l}150-905 \mathrm{~A}- \\
64 \mathrm{X}-5,65-68 \text { (treated) } \\
64 \mathrm{X}-5,65-68^{\mathrm{a}}\end{array}$ & $\begin{array}{l}574.90 \\
574.90\end{array}$ & $\begin{array}{l}\text { Dolomite } \\
\text { Dolomite }\end{array}$ & $\begin{array}{l}4.5 \\
3.5\end{array}$ & $\begin{array}{l}5.4 \\
5.9\end{array}$ & $\begin{array}{l}4.6 \\
5.1\end{array}$ \\
\hline $\begin{array}{l}150-906 \mathrm{~A}- \\
5 \mathrm{H}-3,145-150 \text { (treated) } \\
5 \mathrm{H}-3,145-150 \text { (untreated) } \\
8 \mathrm{X}-3,53-55 \\
10 \mathrm{X}-7,21-24 \\
11 \mathrm{X}-5,55-58 \\
29 \mathrm{X}-6,17-20 \\
36 \mathrm{X}-2,145-147 \\
42 \mathrm{X}-2,6-13 \\
54 \mathrm{X}-5,49-63\end{array}$ & $\begin{array}{r}41.97 \\
41.97 \\
66.03 \\
90.61 \\
98.05 \\
272.77 \\
336.15 \\
392.33 \\
512.69\end{array}$ & $\begin{array}{l}\text { Dolomite } \\
\text { Dolomite/calcite } \\
\text { Siderite } \\
\text { Dolomite } \\
\text { Siderite } \\
\text { Siderite } \\
\text { Dolomite } \\
\text { Dolomite } \\
\text { Siderite }\end{array}$ & $\begin{array}{r}-2.1 \\
-2.0 \\
5.0 \\
-11.4 \\
2.3 \\
11.3 \\
-10.3 \\
-12.0 \\
-1.0\end{array}$ & $\begin{array}{r}-6.4 \\
-6.8 \\
5.3 \\
4.4 \\
7.5 \\
3.4 \\
0.5 \\
0.1 \\
0.1\end{array}$ & $\begin{array}{r}-7.2 \\
-7.6 \\
3.9 \\
3.6 \\
6.1 \\
2.0 \\
-0.3 \\
-0.7 \\
-1.4\end{array}$ \\
\hline
\end{tabular}

Note: Uncorrected $\delta^{18} \mathrm{O}$ values were calculated using a calcite fractionation factor of 1.01020 . The $\delta^{18} \mathrm{O}$ values of siderite and dolomite were corrected by subtracting $1.45 \%$ o Carothers et al., 1988) and $0.8 \%$ (Sharma and Clayton, 1965), respectively. uncorr, $=$ uncorrected $\delta^{18} \mathrm{O}$; corr. $=$ corrected $\delta^{18} \mathrm{O}$; treated $=\mathrm{NaOAc}$ removal of calcite.

${ }^{a}$ Isotopic compositions measured at the Univerity of Tasmania.

an early burial origin or than can be explained by recrystallization at the higher temperatures of deeper burial (Mozley and Burns, 1993). The source of these anomalously low $\delta^{18} \mathrm{O}$ values is uncertain but may include the introduction of meteoric water (Sass and Kolodny, 1972 ) or alteration of pore-water $\delta^{18} \mathrm{O}$ during burial diagenesis.

\section{Calcite}

Most diagenetic carbonates that form in the sulfate-reduction zone have $\delta^{13} \mathrm{C}$ values that range from $-20 \%$ o to $0 \%$ (PDB) (Baker and Burns, 1985). The extreme negative $\delta^{13} \mathrm{C}$ values of some of the calcite cements ( $-37 \%$ o to $-22 \%$ PDB $)$ indicate that they may have derived some of their carbonate from the oxidation of methane. Methane has highly depleted carbon isotopic compositions of $-70 \%$ o to $-40 \%$ (PDB) (Claypool and Kaplan, 1974) and the New Jersey Margin sediments contain abundant methane (Mountain, Miller, Blum, et al., 1994). Oxidation of methane is thought to occur when it migrates into the overlying sulfate-reduction zone and oxic environments (Iversen and Jørgensen, 1985; Paull et al., 1992). For example, $\delta^{13} \mathrm{C}$ values as light as $-32 \%$ o have been reported for diagenetic carbonates at the base of the sulfate-reduction zone (Claypool and Threlkeld, 1983) where the anaerobic oxidation of methane,

$$
\mathrm{SO}_{4}{ }^{2-}+\mathrm{CH}_{4}=\mathrm{HCO}_{3}{ }^{-}+\mathrm{HS}^{-}+\mathrm{H}_{2} \mathrm{O} \text {, }
$$

may have been an important source of isotopically light bicarbonate. The above reaction results in an increase in carbonate alkalinity and 


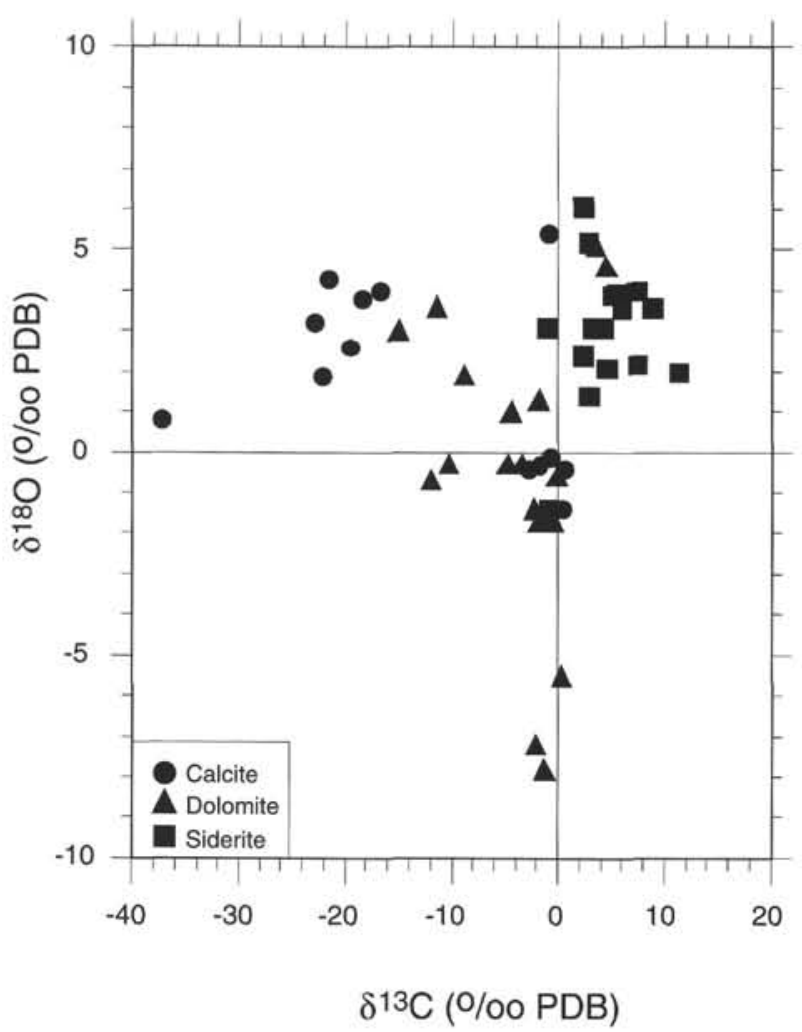

Figure 5. Plot of oxygen and carbon isotopic compositions of diagenetic carbonates from Leg 150. Siderite and dolomite oxygen isotopic values were determined using a fractionation factor of 1.01165 and 1.01100 , respectively (Carothers et al., 1988; Sharma and Clayton, 1965).

has been suggested as a driving mechanism for precipitation of carbonate cements found in association with methane-rich seeps in accretionary prisms (e.g., Ritger et al., 1987; Paull et al., 1992). With extensive local anaerobic methane oxidation, the $\delta^{13} \mathrm{C}$ values of both precipitated carbonate and pore-water $\mathrm{CO}_{2}$ can be very low $(<-28 \%$ o PDB) (Paull et al., 1992).

Methane can also be oxidized in oxic environments near the sediment/seawater interface. Calcite precipitation is favored over dolomite at or near the sediment/seawater interface where the $\mathrm{Mg} / \mathrm{Ca}$ pore-water ratio is low and the sulfate concentration is high. Methane-derived carbonate from the Florida escarpment is low-Mg calcite (Paull et al., 1992). However, methane-derived carbonates from the North Sea are composed of aragonite and $\mathrm{Mg}$ calcite (Hovland et al., 1987). Therefore, calcite cements from this study may have originally been low-Mg calcite or were later recrystallized to low-Mg calcite. Not all calcite nodules of the New Jersey continental slope have low $\delta^{13} \mathrm{C}$ isotopic values (Fig. 7). For example, calcite cemented samples from the upper Oligocene sediment at Site 903 recovered from between 974 and $1110 \mathrm{mbsf}$ have $\delta^{13} \mathrm{C}$ values that range from $0.6 \%$ to $-2.8 \%$ (PDB). Why calcite formed rather than dolomite or siderite in these sediments is unclear. The $\delta^{18} \mathrm{O}$ values of the calcite from Site 903 indicate formation at normal bottom-water and burial temperatures (Fig. 6).

\section{${ }^{87} \mathrm{Sr} /{ }^{86} \mathrm{Sr}$-DERIVED AGES OF THE DIAGENETIC CARBONATES}

The ${ }^{87} \mathrm{Sr} /{ }^{86} \mathrm{Sr}$ ratio of seawater is thought to be uniform throughout the ocean because the residence time of $\mathrm{Sr}$ is longer than the oceanic

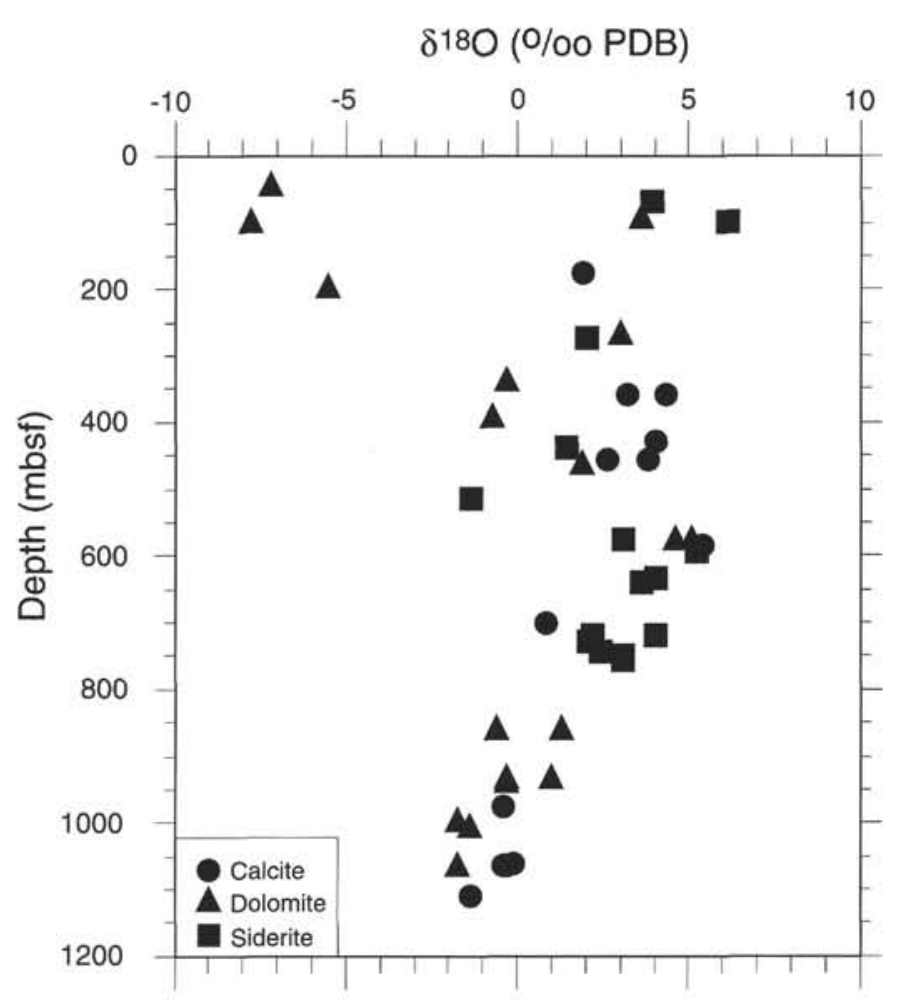

Figure 6. Oxygen isotopic compositions of diagenetic carbonates as a function of sediment depth for Sites 902, 903, and 906. Siderite and dolomite oxygen isotopic values were determined using a fractionation factor of 1.01165 and 1.01100, respectively (Carothers et al., 1988; Sharma and Clayton, 1965). The anomalously high $\delta^{18} \mathrm{O}$ values of diagenetic carbonate samples from below approximately $550 \mathrm{mbsf}$ reflects the $150-300 \mathrm{~m}$ greater thickness of upper Pleistocene sediment at Site 903 (Fig. 2).

mixing time (Broecker and Peng, 1982). The large increase in the seawater ${ }^{87} \mathrm{Sr} /{ }^{86} \mathrm{Sr}$ ratio $(\sim 0.0013)$ from the late Eocene to Holocene makes it possible to use $\mathrm{Sr}$-isotope stratigraphy for correlation and age dating of Sr-bearing marine minerals (Burke et al., 1982; DePaolo and Ingram, 1985; Hess et al., 1986). The ${ }^{87} \mathrm{Sr} /{ }^{86} \mathrm{Sr}$-derived ages of diagenetic carbonates will depend on the source of the Sr. The most significant $\mathrm{Sr}$ sources are $\mathrm{Sr}$ in precursor carbonate and $\mathrm{Sr}$ in pore water originally buried with the sediment. These two $\mathrm{Sr}$ sources should have similar ${ }^{87} \mathrm{Sr} /{ }^{86} \mathrm{Sr}$ ratios if the precursor carbonate fossils and the sediment are the same age (Richter and DePaolo, 1987). The $\mathrm{Sr}$ content of diagenetic carbonates is expected to be less than the $\mathrm{Sr}$ content of the precursor carbonate. No isotopic fractionation is expected during formation of the diagenetic carbonates because of the small difference in mass between ${ }^{87} \mathrm{Sr}$ and ${ }^{86} \mathrm{Sr}$. Therefore, we anticipate that the ${ }^{87} \mathrm{Sr} /{ }^{86} \mathrm{Sr}$ ratio of the diagenetic carbonate should reflect the ratio of the sediment in which it formed.

The Sr-derived ages of diagenetic carbonates are highly variable but generally become younger upsection at Sites 902, 903, and 906 (Table 5; Fig. 12). The unreasonably young ages at Sites 902 and 903 may be explained by leaching of radiogenic ${ }^{87} \mathrm{Sr}$ from terrigenous detrital material either during sample processing or during burial diagenesis. The presence of $\mathrm{Al}$ and $\mathrm{K}$ in the elemental analyses of the acid-soluble fraction (Table 1) indicates that minor solution of noncarbonate minerals generally increases with decreasing carbonate content of the bulk samples (Table 1; Fig. 4). Therefore, reaction with similar strength acid $(1.5 \mathrm{~N} \mathrm{HCl})$ for $\mathrm{Sr}$-isotopic analysis may have leached $\mathrm{Sr}$ from ${ }^{87} \mathrm{Sr}$-rich detrital clay minerals. The low $\mathrm{Sr}$ contents of the diagenetic carbonates, especially the siderites, require only minor amounts of leached terrigenous $\mathrm{Sr}$ to impact the ${ }^{87} \mathrm{Sr} /{ }^{86} \mathrm{Sr}$ ratio. For example, the unreasonably young age of the calcite cement from 


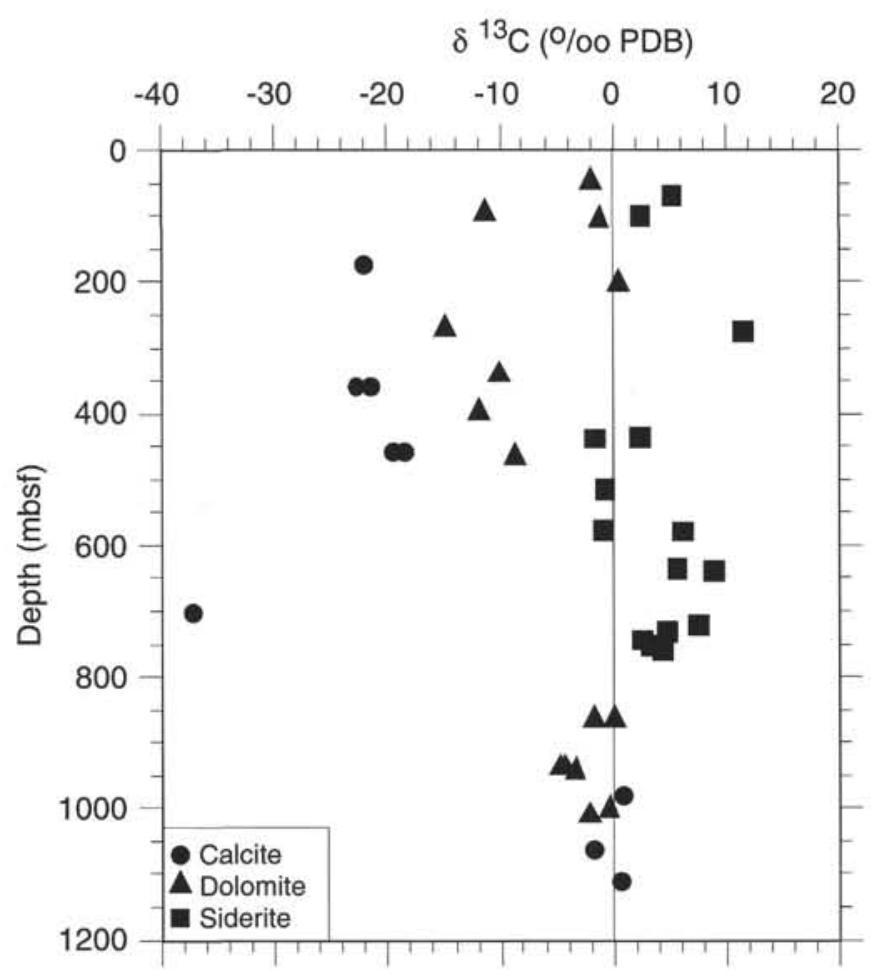

Figure 7. Carbon isotopic composition as a function of sediment depth for Sites 902,903 , and 906 .

Sample $150-903 \mathrm{C}-41 \mathrm{R}-5,80-83 \mathrm{~cm}$, is probably a result of the low calcite content of the bulk sample $(7.5 \mathrm{wt} \%)$ and the low $\mathrm{Sr}$ content of the calcite cement of $170 \mathrm{ppm}$.

At Sites 902,903 , and 906, most of the carbonates are older than the surrounding sediment. A possible explanation is that older diagenetic carbonate or, more likely, their biogenic precursors, as the nodules and cements appear to have formed in place, were reworked and transported by way of debris flows into younger sediment. Numerous debris flows and reworked shallow-water benthic foraminifers indicate increased deposition of reworked sediment in the middle and upper Miocene sediments at these sites (Mountain, Miller, Blum, et al., 1994). Another possible mechanism to explain carbonate cements older than the surrounding sediments is diffusion of $\mathrm{Sr}$ from older underlying sediments. Pore-water $\mathrm{Sr}$ concentrations increase with depth at these sites (Fig. 9) and indicate that $\mathrm{Sr}$ is currently diffusing upward from the underlying Eocene carbonates. Upward diffusion of $\mathrm{Sr}$ should cause age differences between carbonate cements and sediment to decrease upsection. The fact that this trend was not observed may indicate that deeply buried carbonates were formed at shallow burial depths and have not recrystallized (Table 5). Therefore, diagenetic carbonates from Sites 902, 903, and 906 apparently do not yield reliable ${ }^{87} \mathrm{Sr} /{ }^{86} \mathrm{Sr}$-derived ages.

\section{RELATION OF CARBONATE CEMENTS TO SEA LEVEL}

The sedimentation rate on the New Jersey Margin was highly variable and dependent on sea-level fluctuations and sediment supply (Mountain, Miller, Blum, et al., 1994). The highly variable sedimentation rates of the New Jersey Margin were a major controlling factor in the precipitation of carbonate minerals. The presence of diagenetic carbonate nodules below the lower boundaries of unconformities suggests that sufficiently long periods of slow or nondeposition en- hanced diagenetic carbonate cementation (McCracken et al., this volume). During periods of slow sedimentation rates, perhaps associated with maximum rate of sea-level rise, dolomite is expected to form in the sulfate-reduction zone (Pisciotto and Mahoney, 1981; Burns and Baker, 1987). Calcite and dolomite from the upper Eocene to lower Miocene of the New Jersey Margin formed in the sulfate-reduction zone and are associated with periods of relatively slow deposition. Middle to late Miocene dolomite precipitated predominantly in the upper portions of the methanogenesis zone in response to generally higher sedimentation rates. Dolomite currently forming in the uppermost 200 mbsf at Site 903 is in the upper portion of the methanogenesis zone of rapidly deposited, organic-rich Pleistocene sediments. These samples have $\delta^{13} \mathrm{C}$ values of about $0 \%$ and are associated with high sedimentation rates of approximately $700 \mathrm{~m} / \mathrm{m}$.y. (Mountain, Miller, Blum, et al., 1994). The increased burial flux of Fe-rich terrigenous material during the middle to late Miocene resulted in abundant siderite formation along with ferroan dolomite. Abundant precipitation of siderite is associated with increased sedimentation rates during the middle to late Miocene as the shelf/slope break migrated seaward and the rate of organic matter and Fe-rich terrigenous sedimentation increased and shoaled the sediment depth to the transition from the sulfate-reduction to the methanogenesis zone.

\section{CONCLUSIONS}

Diagenetic carbonate minerals from the upper slope of the New Jersey Margin are a byproduct of organic matter degradation and the dissolution of precursor biogenic calcite, mostly foraminifers and nannofossils. Pore-water chemistry, biogenic calcite precursors, rate of organic-carbon degradation and sedimentation rate are important factors that control the mineralogy and abundance of the diagenetic carbonates. Dolomite formation within the sulfate-reduction zone and upper methanogenesis zone was favored by high alkalinity, low sulfate ion concentrations, and high $\mathrm{Mg} / \mathrm{Ca}$ ratios. Dolomite that formed in the methanogenesis zone contains more Fe than dolomite that formed in the overlying sulfate-reduction zone, because Fe was no longer preferentially removed by pyrite. The high Fe content of the terrigenous-rich sediment increased the pore-water $\mathrm{Fe} / \mathrm{Mg}$ ratio and favored siderite precipitation over dolomite in the zone of methanogenesis. Diagenetic calcite formed in the sulfate-reduction zone, and as cements where methane seeps intersected overlying sulfatereduction or suboxic/oxic zones.

Highly variable sedimentation rates on the New Jersey Margin appear to have had a large influence on the abundance and mineralogy of diagenetic minerals precipitated. During periods of slow sedimentation rates, perhaps associated with maximum rates of sea-level rise, dolomite precipitation was favored in the sulfate-reduction zone. More extensive cementation was possible due to the longer residence time near the sediment/seawater interface. During periods of high sedimentation rates, dolomite and siderite precipitation occurred predominantly in the zone of methanogenesis as less tightly cemented nodules and layers.

The Sr-derived ages of diagenetic carbonates from Sites 902, 903 , and 906 are highly variable and generally become younger upsection. Possible reasons for discrepancies between the age of carbonate cements and the surrounding sediment include leaching of ${ }^{87} \mathrm{Sr}$ from noncarbonate minerals during acid digestion, diffusion of $\mathrm{Sr}$ from underlying Eocene limestones, and reworking of older biogenic precursors or diagenetic carbonate into younger sediments.

\section{ACKNOWLEDGMENTS}

We thank the members of the Leg 150 scientific party, technicians, and crew. W. Sackett assisted with stable isotope analyses at 
Table 4. Interstitial water data for Site 903.

\begin{tabular}{|c|c|c|c|c|c|c|c|c|c|c|c|c|c|c|}
\hline $\begin{array}{l}\text { Core, section, } \\
\text { interval }(\mathrm{cm})\end{array}$ & $\begin{array}{l}\text { Depth } \\
\text { (mbsf) }\end{array}$ & $\mathrm{pH}$ & $\begin{array}{l}\text { Alk. } \\
(\mathrm{mM})\end{array}$ & $\begin{array}{l}\text { Sal. } \\
(\% c)\end{array}$ & $\begin{array}{l}\mathrm{Cl} \text { (tit) } \\
(\mathrm{mM})\end{array}$ & $\begin{array}{c}\mathrm{SO}_{4} \\
(\mathrm{mM})\end{array}$ & $\underset{(\mathrm{mM})}{\mathrm{NH}_{4}}$ & $\underset{(\mathrm{mM})}{\mathrm{PO}_{4}}$ & $\begin{array}{c}\mathrm{Sr} \\
(\mu \mathrm{M})\end{array}$ & $\begin{array}{l}\mathrm{Ca} \text { (tit) } \\
(\mathrm{mM})\end{array}$ & $\begin{array}{c}\mathrm{Mg}(\mathrm{tit}) \\
(\mathrm{mM})\end{array}$ & $\mathrm{Mg} / \mathrm{Ca}$ & $\begin{array}{c}\mathrm{Fe}(\mathrm{AA}) \\
(\mu \mathrm{M})\end{array}$ & $\begin{array}{c}\mathrm{Mn}(\mathrm{AA}) \\
(\mu \mathrm{M})\end{array}$ \\
\hline \multicolumn{15}{|l|}{$150-903 \mathrm{~A}-$} \\
\hline $1 \mathrm{H}-1,145-150$ & 1.5 & 7.5 & 7.9 & 35.5 & 564 & 25.5 & 0.3 & 36 & 78 & 9.7 & 52.7 & 5.4 & 6 & 4 \\
\hline $2 \mathrm{H}-1,145-150$ & 14.0 & 7.4 & 16.0 & 33.5 & 565 & 0.2 & 3.5 & 115 & 65 & 3.6 & 39.6 & 11.0 & 27 & 2 \\
\hline $3 \mathrm{H}-3,145-150$ & 23.5 & 7.4 & 12.1 & 33.2 & 560 & 0.0 & 4.6 & 62 & 67 & 4.0 & 37.6 & 9.5 & NM & 2 \\
\hline $4 \mathrm{H}-4,145-150$ & 34.5 & 7.4 & 10.3 & 33.8 & 562 & 0.3 & 4.7 & 24 & 71 & 4.4 & 37.1 & 8.4 & 49 & 3 \\
\hline $5 \mathrm{H}-3,145-150$ & 42.5 & 7.5 & 7.7 & 33.0 & 565 & 0.4 & 6.5 & 29 & 72 & 4.3 & 35.3 & 8.2 & 50 & 3 \\
\hline $6 \mathrm{H}-3,145-150$ & 52.0 & 7.5 & 7.6 & 33.5 & 566 & 0.2 & 5.2 & 24 & 72 & 4.3 & 33.9 & 7.9 & 54 & 3 \\
\hline $7 \mathrm{H}-3,145-150$ & 61.5 & 7.5 & 7.8 & 33.5 & 563 & 0.1 & 4.9 & 19 & 72 & 4.4 & 34.4 & 7.8 & 45 & 3 \\
\hline $8 \mathrm{H}-3,145-150$ & 71.0 & 7.5 & 6.3 & 33.0 & 564 & 0.0 & 4.3 & 10 & 72 & 4.7 & 33.5 & 7.1 & 44 & 3 \\
\hline $9 \mathrm{H}-3,145-150$ & 80.5 & 7.5 & 6.6 & 33.5 & 560 & 0.2 & 4.5 & 13 & 70 & 4.8 & 33.4 & 6.9 & 44 & 3 \\
\hline $10 \mathrm{H}-3,150-155$ & 90.0 & 7.5 & 6.9 & 33.0 & 559 & 0.5 & 5.2 & 13 & 67 & 4.3 & 32.2 & 7.5 & 37 & 3 \\
\hline $11 \mathrm{H}-3,145-150$ & 99.5 & 7.6 & 10.6 & 33.0 & 557 & 0.3 & 7.3 & 37 & 62 & 4.0 & 31.3 & 7.9 & 14 & 3 \\
\hline $12 \mathrm{H}-3,145-150$ & 109.0 & 7.5 & 13.1 & 33.0 & 558 & 0.7 & 9.4 & 62 & 60 & 3.8 & 31.3 & 8.3 & 24 & 2 \\
\hline $14 \mathrm{H}-3,143-150$ & 128.0 & 7.7 & 18.0 & 33.5 & 558 & 0.3 & 11.3 & 36 & 58 & 4.1 & 32.5 & 8.0 & 2 & NM \\
\hline $17 \mathrm{H}-2,140-150$ & 152.0 & 7.8 & 18.0 & 33.0 & 565 & 0.3 & 8.7 & 23 & 52 & 3.3 & 28.6 & 8.8 & 3 & 1 \\
\hline $21 \mathrm{X}-2,145-150$ & 172.5 & 7.6 & 12.3 & 33.0 & 556 & 0.4 & 10.4 & 50 & 59 & 3.7 & 27.8 & 7.6 & 10 & 2 \\
\hline $24 \mathrm{X}-3,145-150$ & 196.4 & 7.6 & 31.8 & 34.5 & 556 & 1.0 & 10.3 & 125 & 68 & 4.4 & 32.8 & 7.5 & 5 & 3 \\
\hline $27 \mathrm{X}-3,140-150$ & 225.1 & 7.8 & 16.5 & 34.0 & 564 & 0.0 & 8.7 & 18 & 59 & 3.9 & 30.1 & 7.6 & 8 & 2 \\
\hline $30 \times-2,140-150$ & 252.7 & 7.7 & 7.5 & 33.5 & 575 & 0.7 & 8.0 & 8 & 67 & 5.1 & 29.1 & 5.7 & NM & 2 \\
\hline $33 \mathrm{X}-3,140-150$ & 283.0 & 7.6 & 14.4 & 35.0 & 581 & 0.3 & 6.8 & 26 & 86 & 6.6 & 31.0 & 4.7 & 9 & 5 \\
\hline $36 \mathrm{X}-3,140-150$ & 312.0 & 7.8 & 10.2 & 35.5 & 598 & 0.3 & 7.5 & 6 & 104 & 6.3 & 31.1 & 5.0 & 6 & 2 \\
\hline $39 \mathrm{X}-3,140-150$ & 340.9 & 7.7 & 12.8 & 36.2 & 618 & 0.0 & 8.6 & 16 & 124 & 7.2 & 33.7 & 4.7 & NM & NM \\
\hline $42 \mathrm{X}-3,140-150$ & 369.9 & 7.3 & 27.9 & 40.0 & 658 & 0.1 & 6.3 & 24 & 149 & 9.4 & 39.9 & 4.2 & 6 & $\mathrm{NM}$ \\
\hline $45 \mathrm{X}-2,140-150$ & 397.3 & 7.1 & 32.5 & 42.0 & 687 & 0.1 & 5.5 & 10 & 154 & 10.7 & 42.2 & 3.9 & 9 & 3 \\
\hline $48 \mathrm{X}-3,140-150$ & 427.8 & 7.7 & 34.4 & 44.0 & 727 & 0.3 & 7,1 & 5 & 154 & 10.0 & 41.7 & 4.2 & NM & 2 \\
\hline $51 X-3,140-150$ & 456.7 & 7.0 & 34.6 & 46.0 & 753 & 0.5 & 7.3 & 11 & 166 & 10.1 & 44.5 & 4.4 & 14 & 2 \\
\hline $54 \mathrm{X}-3,140-150$ & 485.8 & 6.9 & 38.6 & 46.5 & 815 & 0.2 & 9.5 & 6 & 203 & 11.8 & 45.7 & 3.9 & 15 & 2 \\
\hline $57 \mathrm{X}-2,140-150$ & 513.2 & 7.0 & 35.6 & 50.0 & 826 & 0.3 & 13.2 & 25 & 222 & $\begin{array}{l}10.8 \\
10.8\end{array}$ & 45.2 & 4.2 & 16 & 3 \\
\hline $60 X-3,140-150$ & 543.7 & 7.6 & 36.3 & 51.0 & 830 & 0.1 & 10.2 & 3 & 236 & $\begin{array}{l}13.4 \\
\end{array}$ & 45.5 & 3.4 & NM & 3 \\
\hline $63 \mathrm{X}-3,140-150$ & 572.3 & 7.5 & 36.0 & 49.0 & 836 & 0.0 & 10.9 & 17 & 253 & 14.4 & 48.0 & 3.3 & NM & 3 \\
\hline $67 \mathrm{X}-3,140-150$ & 610.8 & 7.7 & 37.7 & 50.0 & 876 & 0.0 & 16.6 & 7 & 253 & 15.2 & 46.9 & 3.1 & NM & 3 \\
\hline $70 \times-3,140-150$ & 639.7 & 6.8 & 35.0 & 53.8 & 883 & 0.0 & 12.3 & 19 & 282 & 16.5 & 48.4 & 2.9 & NM & 4 \\
\hline $73 \times-3,140-150$ & 668.6 & 6.8 & 36.7 & 54.5 & 908 & 0.0 & 13.2 & 3 & 297 & 17.2 & 49.4 & 2.9 & 21 & 3 \\
\hline $76 \mathrm{X}-3,140-150$ & 697.6 & 6.8 & 36.4 & 54.5 & 908 & 0.0 & 12.8 & 21 & 274 & 17.3 & 48.4 & 2.8 & 31 & 3 \\
\hline \multicolumn{15}{|l|}{$150-903 \mathrm{C}-$} \\
\hline $5 \mathrm{R}-1,140-150$ & 507.1 & 6.9 & 38.0 & 50.0 & 847 & 0.0 & 11.3 & 17 & 216 & 14.1 & 47.2 & 3.3 & NM & NM \\
\hline $9 \mathrm{R}-1,140-150$ & 588.0 & 6.9 & 37.0 & 50.5 & 848 & 0.2 & 11.5 & 5 & 248 & 15.1 & 48.5 & 3.2 & 72 & 2 \\
\hline $12 \mathrm{R}-3,140-150$ & 693.1 & 6.7 & 35.2 & 54.0 & 885 & 2.5 & 12.5 & 15 & 310 & 17.8 & 48.9 & 2.7 & NM & 3 \\
\hline $15 \mathrm{R}-2,140-150$ & 720.2 & 6.8 & 34.2 & 54.5 & 897 & 2.9 & 15.5 & 0 & 329 & 18.4 & 47.9 & 2.6 & 75 & 2 \\
\hline $18 \mathrm{R}-1,53-63$ & 747.1 & 6.7 & 32.7 & 54.2 & 896 & 0.8 & 15.8 & 0 & 276 & 17.8 & 46.3 & 2.6 & 32 & 2 \\
\hline $21 \mathrm{R}-1,98-108$ & 776.5 & $\begin{array}{l}0.1 \\
6.8\end{array}$ & 31.7 & 54.0 & 886 & $\begin{array}{l}0.8 \\
1.7\end{array}$ & $\begin{array}{l}13.0 \\
13.0\end{array}$ & 5 & 347 & $\begin{array}{l}19.1 \\
19.1\end{array}$ & $\begin{array}{l}40.3 \\
47.8\end{array}$ & 2.5 & 23 & 3 \\
\hline $25 \mathrm{R}-2,140-150$ & 816.8 & 6.8 & 30.3 & 56.0 & 933 & 0.5 & 12.1 & 5 & 433 & 21.4 & 47.5 & 2.2 & 50 & 3 \\
\hline $29 \mathrm{R}-3,140-150$ & 856.9 & 6.6 & 27.0 & 56.5 & 941 & 0.0 & 12.8 & 5 & 519 & 22.4 & 45.6 & 2.0 & 24 & 2 \\
\hline $32 \mathrm{R}-1,140-150$ & 882.9 & 6.7 & 27.7 & 57.8 & 963 & 0.3 & 14.9 & 0 & 607 & 24.7 & 45.8 & 1.8 & 12 & 2 \\
\hline $35 \mathrm{R}-3,140-150$ & 914.9 & 6.6 & 25.9 & 57.8 & 956 & 0.4 & 12.4 & 5 & 695 & 26.9 & 45.7 & 1.7 & 59 & 2 \\
\hline $38 \mathrm{R}-1,105-115$ & 940.1 & 6.6 & 22.7 & 57.8 & 975 & 0.1 & 13.7 & 5 & 763 & 27.3 & 43.0 & 1.6 & 21 & 1 \\
\hline $41 \mathrm{R}-3,140-150$ & 972.0 & 6.6 & 22.8 & 57.8 & 958 & 0.9 & 12.6 & 3 & 778 & 28.4 & 44.7 & 1.6 & 32 & 2 \\
\hline $47 \mathrm{R}-2,142-152$ & 1028.5 & 6.7 & 19.3 & 56.2 & 945 & 1.0 & 10.9 & 0 & 849 & 28.5 & 44.6 & 1.6 & NM & 2 \\
\hline $51 \mathrm{R}-3,140-150$ & 1058.9 & NM & NM & NM & 947 & NM & 0.0 & NM & NM & $\mathrm{NM}$ & NM & NM & NM & NM \\
\hline $54 \mathrm{R}-4,140-150$ & 1089.4 & NM & NM & NM & 986 & 0.0 & 0.0 & 0 & NM & 33.3 & 47.2 & 1.4 & NM & NM \\
\hline $57 \mathrm{R}-1,140-150$ & 1113.0 & NM & $\mathrm{NM}$ & 55.8 & 935 & NM & 6.4 & 0 & 1138 & 27.7 & 48.4 & 1.7 & NM & NM \\
\hline
\end{tabular}

Note: $\mathrm{NM}=$ not measured, tit $=$ titration, and $\mathrm{AA}=$ atomic absorption.

USF, K. Miller and M. Feigenson provided the Sr-isotope data, and T. Greco assisted with the SEM work. This study was supported by JOI/USSAC. We thank J. Hein, K. Miller, R. Byrne, and an anonymous reviewer for comments and suggestions that improved the manuscript.

\section{REFERENCES}

Anovitz, L.M., and Essene, E.J., 1987. Phase equilibria in the system $\mathrm{CaCO}_{3}$ $\mathrm{MgCO}_{3}-\mathrm{FeCO}_{3}$. J. Petrol., 28:389-414.

Baker, P.A., and Burns, S.J., 1985. The occurrence and formation of dolomite in organic-rich continental margin sediments. AAPG Bull., 69:1917-1930.

Baker, P.A., and Kastner, M., 1981. Constraints on the formation of sedimentary dolomite. Science, 213:215-216.

Berggren, W.A., Kent, D.V., Flynn, J.J., and Van Couvering, J.A., 1985. Cenozoic geochronology. Geol. Soc. Am. Bull., 96:1407-1418.

Berner, R.A., 1980. Early Diagenesis: A Theoretical Approach: Princeton, NJ (Princeton Univ. Press).

, 1981. A new geochemical classification of sedimentary environments. J. Sediment. Petrol., 51:359-365.

, 1984. Sedimentary pyrite formation: an update. Geochim. Cosmochim. Acta, 48:605-615.
Broecker, W.S., and Peng, T.-H., 1982. Tracers in the Sea: Palisades, NY (Lamont-Doherty Geol, Observ.).

Burke, W.H., Denison, R.E., Hetherington, E.A., Koepnick, R.B., Nelson, H.F., and Otto, J.B., 1982. Variation of seawater ${ }^{87} \mathrm{Sr} /{ }^{86} \mathrm{Sr}$ throughout Phanerozoic time. Geology, 10:516-519.

Burns, S.J., and Baker, P.A., 1987. A geochemical study of dolomite in the Monterey formation, California. J. Sediment. Petrol., 57:128-139.

Carothers, W.W., Adami, L.H., and Rosenbauer, R.J., 1988. Experimental oxygen isotope fractionation between siderite-water and phosphoric acid liberated $\mathrm{CO}_{2}$-siderite. Geochim. Cosmochim. Acta, 52:2445-2450.

Claypool, G.E., and Kaplan, I.R., 1974. The origin and distribution of methane in marine sediments. In Kaplan, I.R. (Ed.), Natural Gases in Marine Sediments: New York (Plenum), 99-139.

Claypool, G.E., and Threlkeld, C.N., 1983. Anoxic diagenesis and methane generation in sediments of the Blake Outer Ridge, Deep Sea Drilling Project Site 533, Leg 76. In Sheridan, R.E., Gradstein, F.M., et al., Init. Repts. DSDP, 76: Washington (U.S. Govt. Printing Office), 391-402.

Coleman, M.L., 1985. Geochemistry of diagenetic non-silicate minerals: kinetic considerations. In Eglinton, G., Curtis, C.D., McKenzie, D.P., and Murchison, D.G. (Eds.), Geochemistry of Buried Sediments: London (The Royal Society), 39-54.

Compton, J.S., 1988a. Sediment composition and precipitation of dolomite and pyrite in the Neogene Monterey and Sisquoc Formations, Santa Maria basin area, California. In Shulka, V., and Baker, P.A. (Eds.), Sedi- 
mentology and Geochemistry of Dolostones. Spec. Publ.-Soc. Econ. Paleontol. Mineral., 43:53-64.

, 1988b. Degree of supersaturation and precipitation of organogenic dolomite. Geology, 16:318-321.

- 1992. Early diagenesis and the origin of diagenetic carbonate in sediment recovered from the Argo Basin, northeastern Indian Ocean (Site 765). In Gradstein, F.M., Ludden, J.N., et al., Proc. ODP, Sci. Results, 123: College Station, TX (Ocean Drilling Program), 77-88.

Compton, J.S., and Siever, R., 1986. Diffusion and mass balance of $\mathrm{Mg}$ during early dolomite formation, Monterey Formation. Geochim. Cosmochim. Acta, 50:125-135.

Curtis, C.D., and Coleman, M.L., 1986. Controls on the precipitation of early diagenetic calcite, dolomite, and siderite concretions in complex depositional sequences. In Gautier, D.L. (Ed.), Roles of Organic Matter in Sediment Diagenesis. Spec. Publ.-Soc. Econ. Paleontol. Mineral., 38:2333.

Curtis, C.D., Pearson, M.J., and Somogyi, A.V., 1975. Mineralogy, chemistry, and origin of concretionary siderite sheet (clay-ironstone band) in the Westphalian of Yorkshire. Mineral. Mag., 40:385-393.

DePaolo, D.J., and Ingram, B.L., 1985. High-resolution stratigraphy with strontium isotopes. Science, 227:938-941.

Fritz, P., and Smith, D.C.W., 1970. The isotopic composition of secondary dolomites. Geochim. Cosmochim. Acta., 34:1161-1173.

Froelich, P.N., Klinkhammer, G.P., Bender, M.L., Luedtke, N.A., Heath, G.R., Cullen, D., Dauphin, P., Hammond, D., Hartman, B., and Maynard, V., 1979. Early oxidation of organic matter in pelagic sediments of the eastern equatorial Atlantic: suboxic diagenesis. Geochim. Cosmochim. Acta, 43:1075-1090.

Gieskes, J.M., 1981. Deep-sea drilling interstitial water studies: implications for chemical alteration of the oceanic crust, layers I and II. In Warme, J.E., Douglas, R.G., and Winterer, E.L. (Eds.), The Deep Sea Drilling Project: A Decade of Progress. Spec. Publ.-Soc. Econ. Paleontol. Mineral., 32:149-167.

1983. The chemistry of interstitial waters of deep-sea sediments: interpretation of deep-sea drilling data. In Riley, J.P., and Chester, R. (Eds.), Chemical Oceanography (Vol. 8): London (Academic), 222-269.

Hein, J.R., and Koski, R.A., 1987. Bacterially mediated diagenetic origin for chert-hosted managanese deposits in the Franciscan Complex, California Coast Ranges. Geology, 15:722-726.

Hess, J.R., Bender, M.L., and Schilling, J.G., 1986. Evolution of the ratio of Strontium-87 to Strontium-86 in seawater from Cretaceous to Present. Science, 231:979-984.

Hovland, M., Talbot, M.R., Qvale, H., Olaussen, S., and Aasberg, L., 1987. Methane-related carbonate cements in pockmarks of the North Sea. $J$. Sediment. Petrol., 57:881-892.

Ireland, B.J., Curtis, C.D., and Whiteman, J.A., 1983. Compositional variation within some glauconites and illites and implications for their stability and origins. Sedimentology, 30:769-786.

Iversen, N., and Jørgensen, B.B., 1985. Anaerobic methane oxidation rates at the sulfate-methane transition in marine sediments from Kattegat and Skagerrak (Denmark). Limnol. Oceanogr., 30:944-955.

Maynard, J.B., 1982. Extension of Berner's "new geochemical classification of sedimentary environments" to ancient sediments. J. Sediment. Petrol., 52:1325-1331.

McCrea, J.M., 1950. On the isotopic chemistry of carbonates and a paleotemperature scale. J. Chem. Phys., 18:849-857.
Miller, K.G., Feigenson, M.D., Kent, D.V., and Olsson, R.K., 1988. Upper Eocene to Oligocene isotope $\left({ }^{87} \mathrm{Sr} /{ }^{86} \mathrm{Sr}, \delta^{18} \mathrm{O}, \delta^{13} \mathrm{C}\right)$ standard section, Deep Sea Drilling Project Site 522. Paleoceanography, 3:223-233.

Moore, S.E., Ferrell, R.E., and Aharon, P., 1992. Diagenetic siderite and other ferroan carbonates in a modern subsiding marsh sequence. J. Sediment. Petrol., 62:357-366.

Mountain, G.S., Miller, K.G., Blum, P., et al., 1994. Proc. ODP, Init. Repts., 150: College Station, TX (Ocean Drilling Program).

Mozley, P.S., and Burns, S.J., 1993. Oxygen and carbon isotopic compositions of marine carbonate concretions: an overview. J. Sediment. Petrol., 63:73-83.

Mozley, P.S., and Carothers, W.W., 1992. Elemental and isotopic compositions of siderite in the Kuparuk Formation, Alaska: effect of microbial activity and water/sediment interaction on early pore-water chemistry. $J$. Sediment. Petrol., 62:681-692.

Odin, G.S., and Matter, A., 1981. De glauconiarum origine. Sedimentology, 28:611-643.

Oslick, J.S., Miller, K.G., Feigenson, M.D., and Wright, J.D., 1994. Oligocene-Miocene strontium isotopes: correlation to a glacioeustatic record. Paleoceanography, 9:427-443.

Paull, C.K., Chanton, J.P., Neumann, A.C., Coston, J.A., Martens, C.S., and Showers, W., 1992. Indicators of methane-derived carbonates and chemosynthetic organic carbon deposits: examples from the Florida Escarpment. Palaios, 7:361-375.

Pisciotto, K.A., and Mahoney, J.J., 1981. Isotopic survey of diagenetic carbonates, Deep Sea Drilling Project, Leg 63. In Yeats, R.S., Haq., B.U., et al., Init. Repts. DSDP, 63: Washington (U.S. Govt. Printing Office), 595-609.

Pye, K., Dickson, J.A.D., Schiavon, N., Coleman, M.L., and Cox, M., 1990. Formation of siderite-Mg-calcite-iron sulfide concretions in intertidal marsh and sandflat sediments, north Norfolk, England. Sedimentology, 37:325-343.

Richter, F.M., and DePaolo, D.J., 1987. Numerical models for diagenesis and the Neogene $\mathrm{Sr}$ isotopic evolution of seawater from DSDP Site 590B. Earth Planet. Sci. Lett., 83:27-38.

Ritger, S., Carson, B., and Suess, E., 1987. Methane-derived authigenic carbonates formed by subduction-induced pore water expulsion along the Oregon/Washington margin. Geol. Soc. Am. Bull., 98:147-156.

Robbie, R.A., Hemingway, B.S., and Fisher, J.R., 1978. Thermodynamic properties of minerals and related substances at $298.15 \mathrm{~K}$ and 1 bar pressure at higher temperatures. U.S. Geol. Surv. Bull., 1452.

Sass, E., and Kolodny, Y., 1965. Stable isotopes, chemistry and petrology of carbonate concretions (Mishash Formation, Israel). Chem. Geol, $10: 261-286$.

Sharma, T., and Clayton, R.N., 1965. Measurement of ${ }^{18} \mathrm{O} /{ }^{16} \mathrm{O}$ ratios of total oxygen in carbonates. Geochim. Cosmochim. Acta, 29:1347-1353.

Spotts, J.H., and Silverman, S.R., 1966. Organic dolomite from Point Fermin, California. Am. Mineral., 51:1144-1155.

Thyne, G.D., and Gwinn, C.J., 1994. Evidence for paleoaquifer from early diagenetic siderite of the Cardium Formation, Alberta, Canada. J. Sediment. Res., 64:726-732.

Date of initial receipt: 27 February 1995

Date of acceptance: 6 December 1995

Ms 150SR-023 
Figure 8. Pore-water alkalinity, sulfate, and $\mathrm{pH}$ as a function of sediment depth at Site 903 . The high $\mathrm{pH}$ values below $450 \mathrm{mbsf}$ are probably an artifact of $\mathrm{CO}_{2}$ degassing during sample recovery.
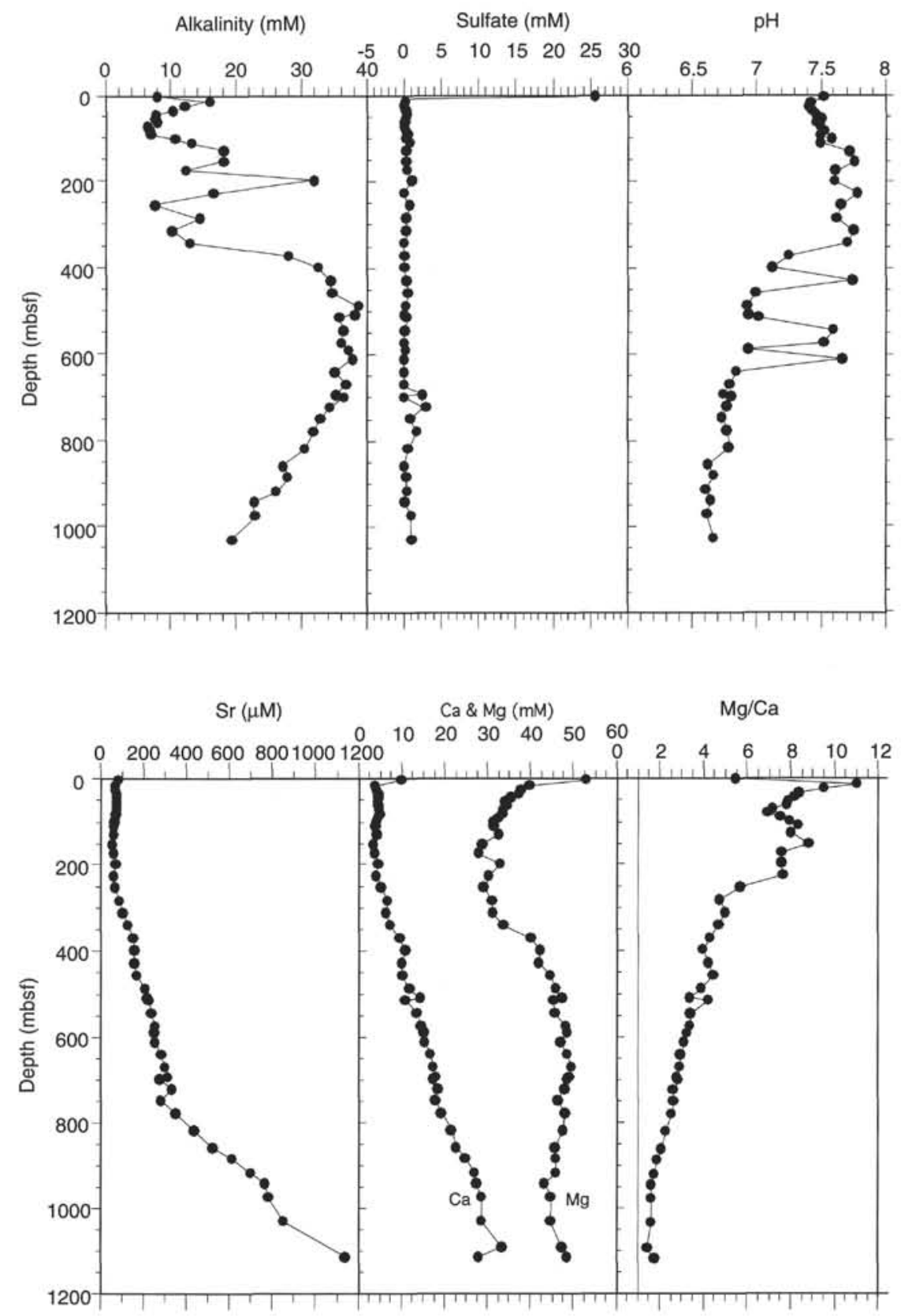


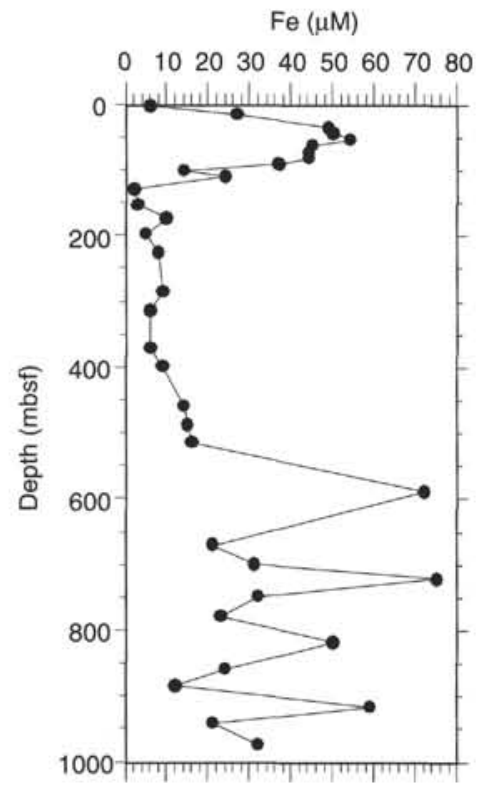

Figure 10. Pore-water Fe concentration as a function of sediment depth at Site 903. The generally high $\mathrm{Fe}$ values but not the exact profile below $500 \mathrm{mbsf}$ were reproducible using DCP.

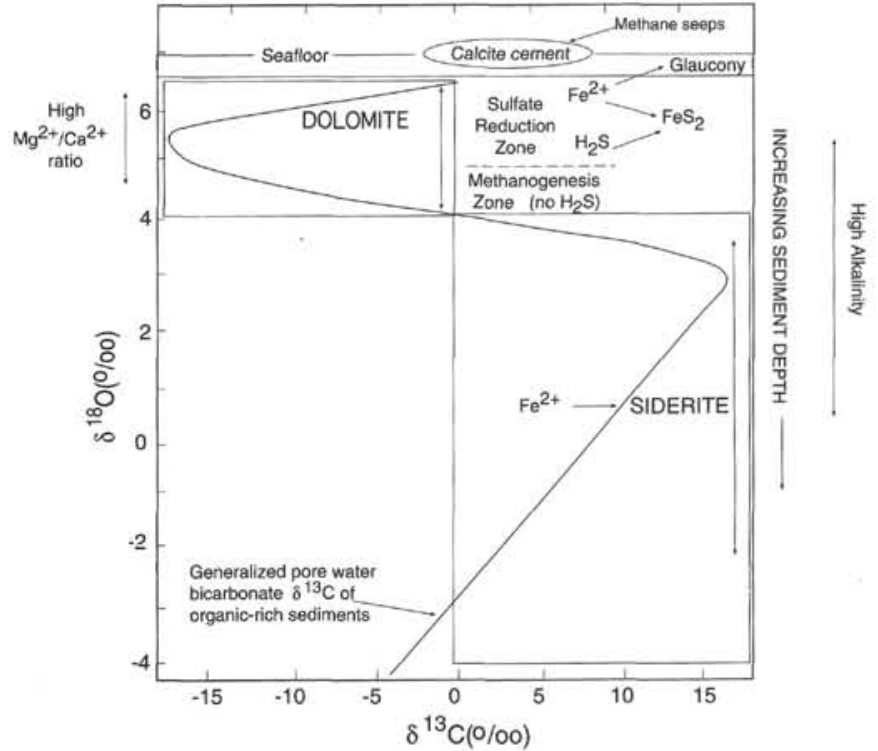

Figure 11. Schematic for the sequence of diagenetic carbonate formation on the New Jersey Margin. The solid line represents generalized pore-water $\delta^{13} \mathrm{C}$ of bicarbonate in organic-rich sediment with increasing burial depth (Claypool and Kaplan, 1974). The $\delta^{18} \mathrm{O}$ axis is for dolomite and calcite; siderite samples that form at equivalent depths will have more negative $\delta^{18} \mathrm{O}$ values. Methane-derived calcite cements may form within the sulfate-reduction zone or near the sediment/seawater interface. Uptake of Fe by glaucony and pyrite restrict most siderite formation to the methanogenesis zone.

Table 5. Sr isotopic results of diagenetic carbonate samples from Leg 150.

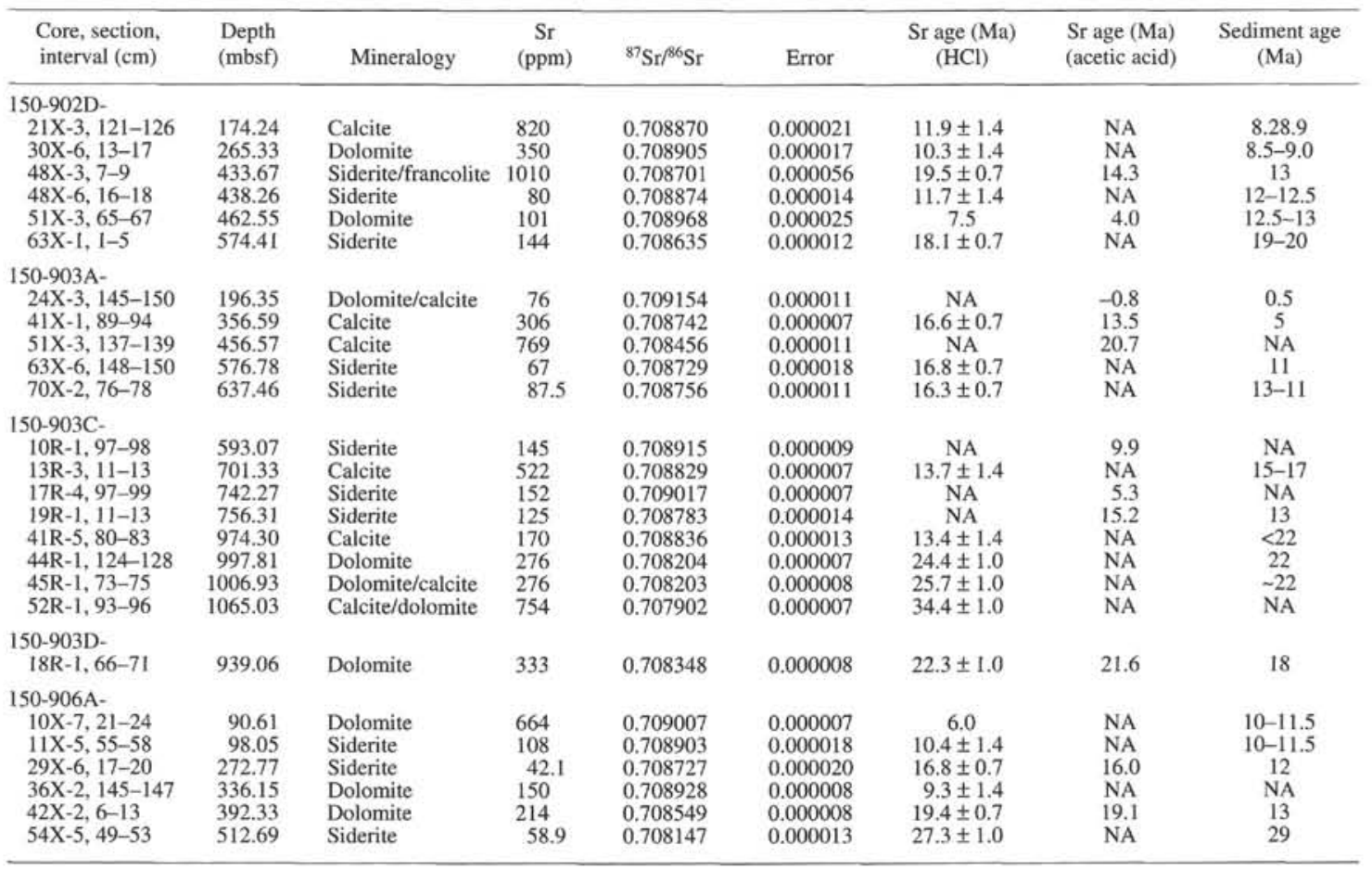

Notes: $\mathrm{HCl}=$ dissolved in $1.5 \mathrm{~N} \mathrm{HCl}$. Acetic acid = dissolved in buffered acetic acid. Sediment age = biostratigraphic ages based on foraminifer assemblages according to Mountain, Miller, Blum, et al. (1994). NA = not analyzed. 


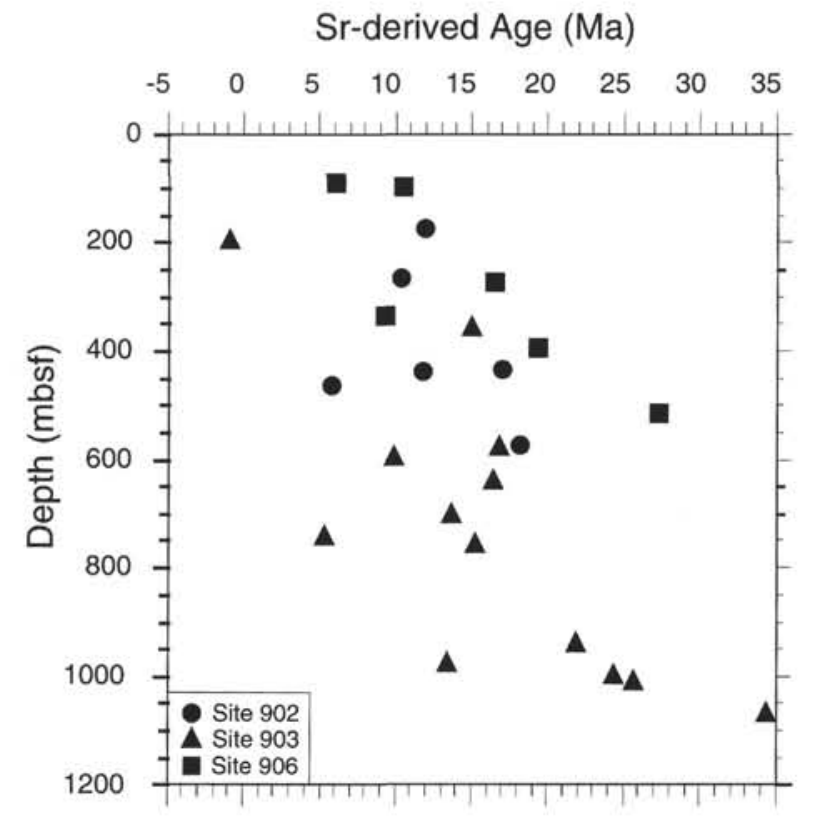

Figure 12. Sr-derived isotopic ages of the diagenetic carbonates as a function of sediment depth for Sites 902, 903, and 906. 

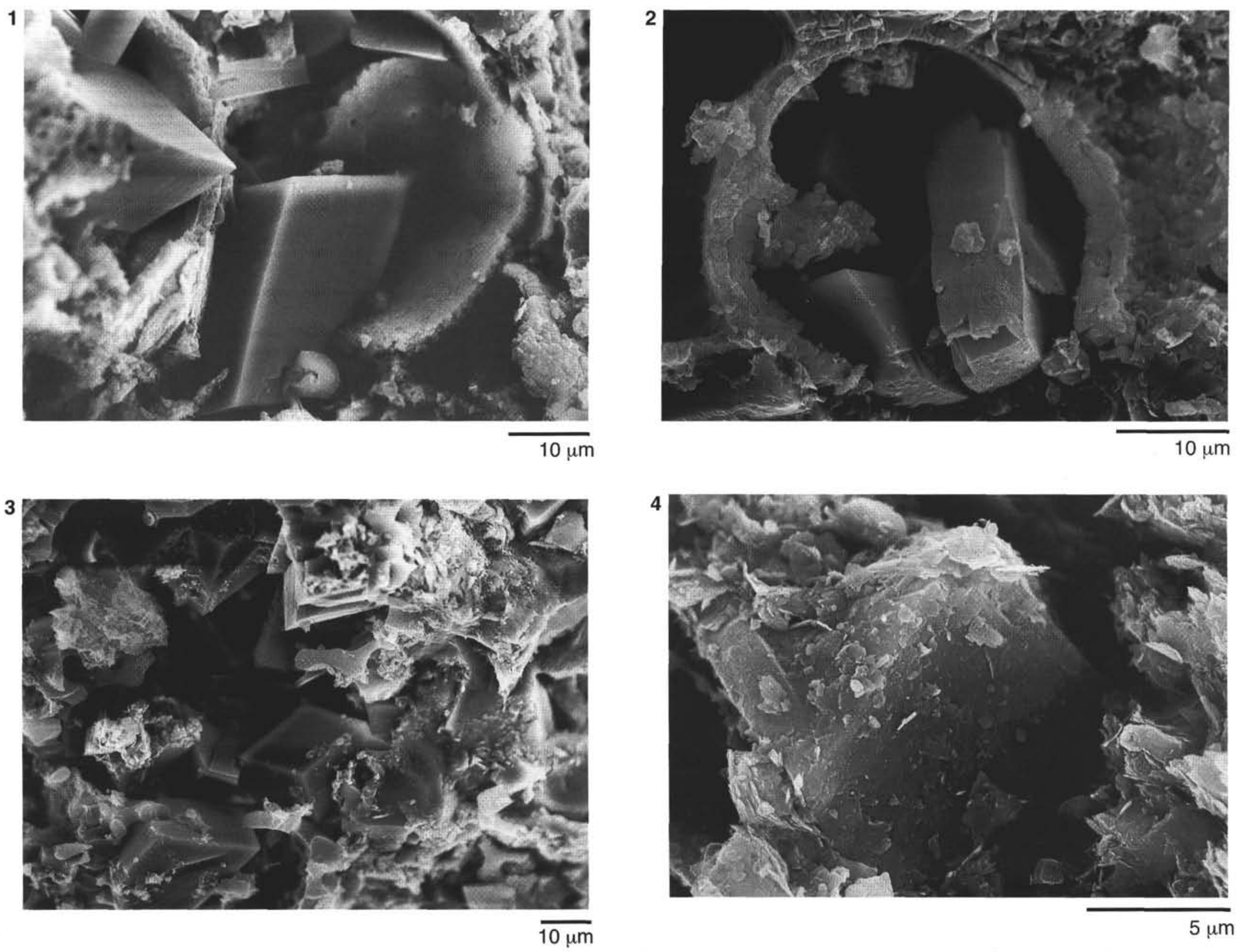

Plate 1. SEM photomicrographs of dolomite rhombs. 1, 2. Sample 150-903D-21R-8, $46-49 \mathrm{~cm}$ (1005.95 mbsf). Single, euhedral dolomite rhomb and associated biogenic calcite. Scale bars $=10 \mu \mathrm{m} .3$. Cluster of dolomite rhombs from Sample 150-903D-21R-8, 46-49 cm (1005.92 mbsf). Scale bar $=10 \mu \mathrm{m}$. 4. Sample 150-903A-11H-3, 145-150 cm. Shallow-burial dolomite/calcite mixture forming in the zone of methanogenesis at $99.45 \mathrm{mbsf}$. Scale bar $=5 \mu \mathrm{m}$. 

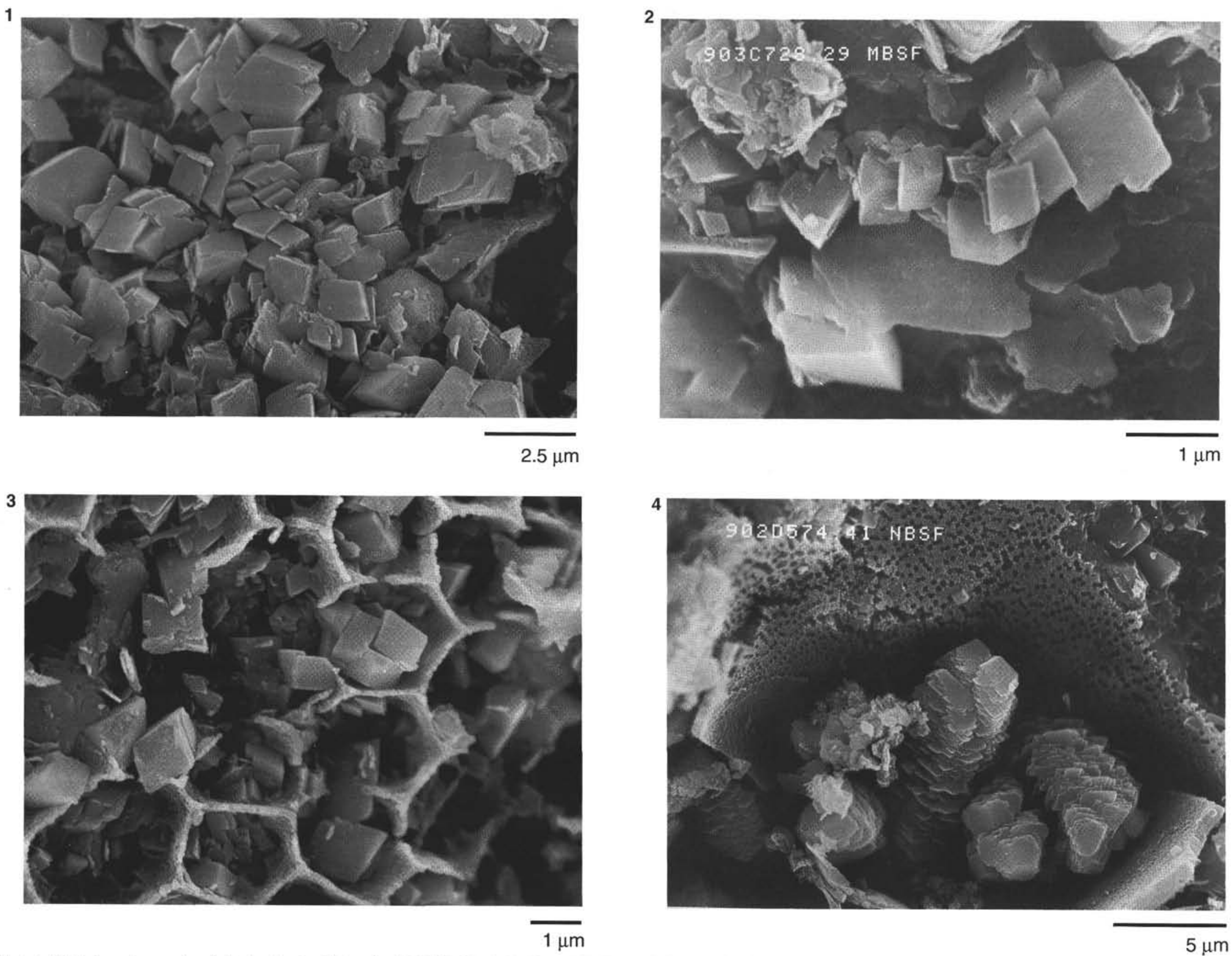

Plate 2. SEM photomicrographs of siderite rhombs. 1. Sample 150-903C-19R-1, 11-13 cm (701.33 mbsf). Cluster of euhedral siderite rhombs. Scale bar $=2.5 \mu \mathrm{m} .2$. Sample $150-903 \mathrm{C}-16 \mathrm{R}-1,129-131 \mathrm{~cm}$ ( $728.29 \mathrm{mbsf})$. Euhedral siderite rhombs. Scale bar $=1 \mu \mathrm{m}$. 3. Sample $150-903 \mathrm{C}-19 \mathrm{R}-1,11-13 \mathrm{~cm}(701.33 \mathrm{mbsf})$. Siderite rhombs infilling the pores of biogenic precursor. Scale bar $=1 \mu \mathrm{m} .4$. Sample $150-$ 902D-63X-1, 1-5 cm (574.41 mbsf). Siderite associated with biogenic calcite. Scale bar $=5 \mu \mathrm{m}$. 